\title{
Preparation of Chiral Ligands Connected with Quaternary Ammonium Group for Recyclable Catalytic Asymmetric Transfer Hydrogenation in Ionic Liquid
}

\author{
Hitomi Uchimoto, ${ }^{a}$ Tomoko Tsuji, ${ }^{b}$ Ikuo Kawasaki, ${ }^{*}, a, b$ Kenji Arimitsu, ${ }^{a}$ Hiroyuki Yasui, ${ }^{c}$ \\ Masayuki Yamashita, ${ }^{b}$ Shunsaku Ohta, ${ }^{b}$ and Kiyoharu Nishide, ${ }^{* a}$ \\ ${ }^{a}$ School of Pharmaceutical Sciences, Mukogawa Women's University; 11-68 Koshien Kyuban-cho, Nishinomiya \\ 663-8179, Japan: ${ }^{b}$ Department of Pharmaceutical Manufacturing Chemistry, Kyoto Pharmaceutical University; 1 \\ Shichono-cho, Misasagi, Yamashina-ku, Kyoto 607-8414, Japan: and ${ }^{c}$ Department of Analytical and Bioinorganic \\ Chemistry, Division of Analytical and Physical Sciences, Kyoto Pharmaceutical University; 5 Nakauchi-cho, \\ Misasagi, Yamashina-ku, Kyoto 607-8414, Japan. \\ Received October 30, 2014; accepted December 11, 2014
}

Reuse of chiral ruthenium catalyst in catalytic asymmetric transfer hydrogenation (CATH) has attracted attention from economic and environmental viewpoints, and reactions using ionic liquids (ILs) as solvent are recognized as one of the most useful methods for reuse of the catalyst. We synthesized $(1 S, 2 S)-N$ ( $p$-toluenesulfonyl)-1,2-diphenylethylenediamine (TSDPEN) derivatives with various ionic moieties, and investigated the effect of their structure with respect to catalytic ability and recyclability in CATH with ILs. Ligand 3a having an imidazolium group showed the best results, and significant differences were observed depending on the structure of the ionic moiety or the length of the alkyl chain connecting the ligand site and the ionic moiety. Among various prochiral ketones used as substrates at various cycles, 3a showed a relatively good result.

Key words ionic liquid; recyclable catalytic asymmetric hydrogenation; task-specific ligand; imidazolium salt

Catalytic asymmetric transfer hydrogenation (CATH) is a very useful method for obtaining optically active secondary alcohols from prochiral ketones and for serving as an alternative to catalytic asymmetric hydrogenation with molecular hydrogen. CATH has many advantages in terms of safety and convenience over conventional hydrogenation because it uses 2-propanol or formic acid as a hydrogen source. ${ }^{1,2)}$ Similar to catalytic asymmetric hydrogenation, CATH can also proceed with high catalytic efficiency and enantioselectivity by using a chiral transition metal complex. Especially well known as a catalyst is the ruthenium complex (1 and 2) with chiral $N$-( $p$-toluenesulfonyl)-1,2-diphenylethylenediamine (TsDPEN) reported by Noyori and colleagues. ${ }^{3-5)} \mathrm{CATH}$ with the ruthenium catalyst has a high degree of applicability for synthetic chemistry in the laboratory as well as in industry. Several attempts to use CATH with recyclable catalysts have been reported with the aim of industrial application. The reuse of expensive transition metals such as ruthenium and the reduction of waste from reactions is advantageous from economic and environmental viewpoints. Indeed, transfer hydrogenation using polymer- or dendritic-supported TsDPEN ligand under heterogeneous conditions has been reported. ${ }^{6-13)}$

Serving as a new type of solvent for green technology are ionic liquids (ILs), which consist of ions such as quaternary ammonium or phosphonium ions and are liquids at room temperature. $^{14-18)}$ Although the toxicity and biodegradability of ILs have not been sufficiently explored, they are known to be non-volatile and non-flammable. ${ }^{14-19)}$ Therefore, the use of ILs offers safety and environmental protection, as well as eliminates the problem of volatilization loss, which occurs with conventional organic solvents. In addition, ILs have the ability to dissolve organic and inorganic compounds, while being immiscible with less polar solvent and water. Organometallic complexes can work as homogeneous catalysts in ILs, and a simple extraction procedure can separate the product from an IL containing a catalyst, which could then be reused for subsequent reactions. Therefore, the use of ILs for organic reactions including transition-metal catalyzed reactions has attracted much attention in the field of green chemistry, and some examples of transfer hydrogenation with IL have been reported. ${ }^{20-25)}$

We have previously reported the synthesis of the taskspecific ionic ligand $\mathbf{3 a}$ and recyclable CATH (RCATH) with $\mathrm{Ru}(\mathrm{II})$ complex coordinated to the chiral ligand 3a using ILs with imidazolium, 1-butyl-3-methyl- $1 H$-imidazolium hexafluorophosphate $\left([\mathrm{bmim}]\left[\mathrm{PF}_{6}\right]\right)$, as a reaction solvent. ${ }^{26)}$ By attaching an imidazolium moiety to TsDPEN, our RCATH showed high reaction efficiency at the 5 th cycle, compared to that using conventional catalyst 2 . From the results, we predicted that $\mathrm{Ru}$ coordinated with ligand $\mathbf{3 a}$ would be immobilized in the IL phase by a cationic moiety such as an imidazolium group. Therefore, we were interested in further details of the effect by the structure of the ligand against the IL phase. Here we describe the synthesis of various TsDPEN derivatives with attached ionic moieties and our examination of RCATH with $\mathrm{Ru}(\mathrm{II})$ complex coordinated with them in IL. We applied recyclable transfer hydrogenation of various ketones at every cycle with the ligand.

\section{Results and Discussion}

For synthesis of TsDPEN derivatives with a variety of ionic moieties, the alkyl chloride $\mathbf{6}$ was first prepared by our method reported previously (Chart 1). Starting from commercially available sodium $p$-hydroxybenzenesulfonate (4), $O$-alkylation 


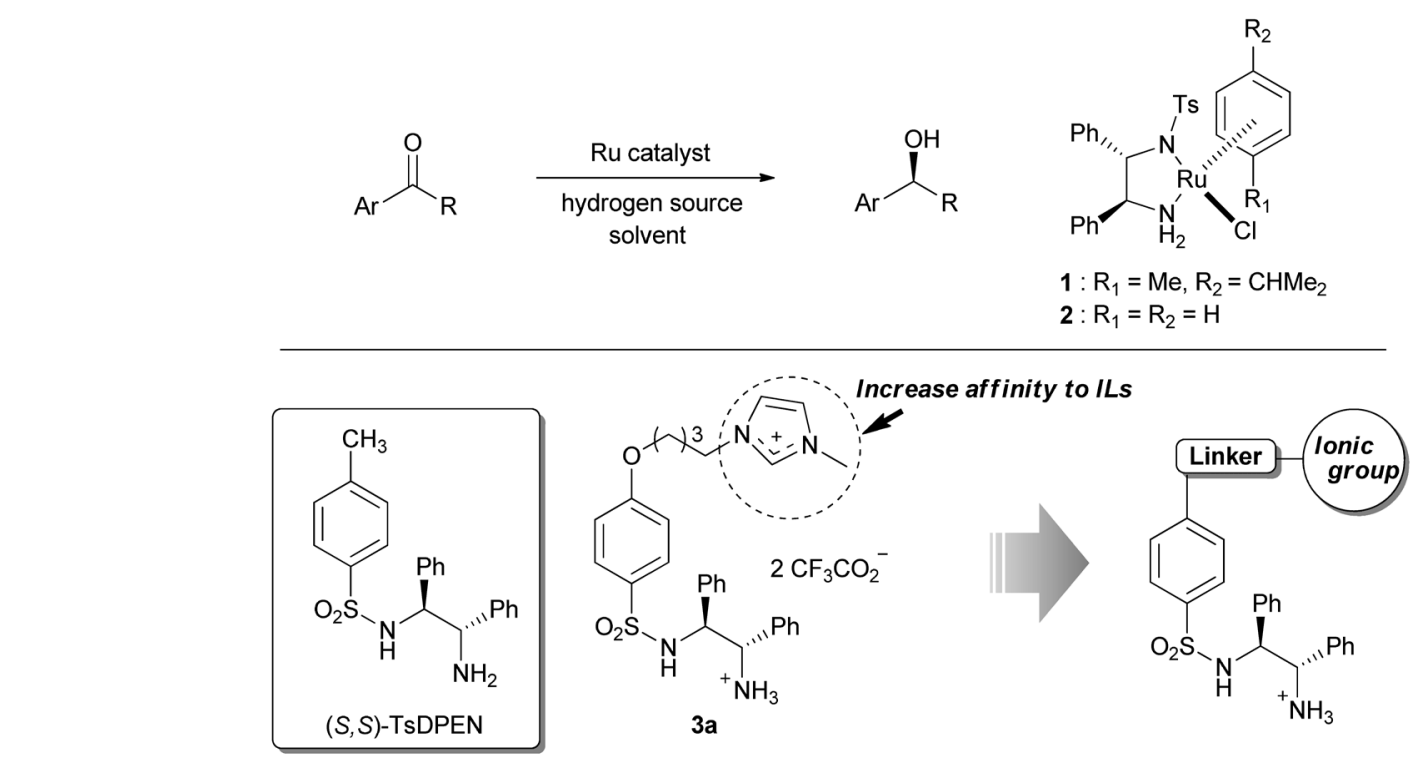

Fig. 1. Design of Chiral Ligands for RCATH

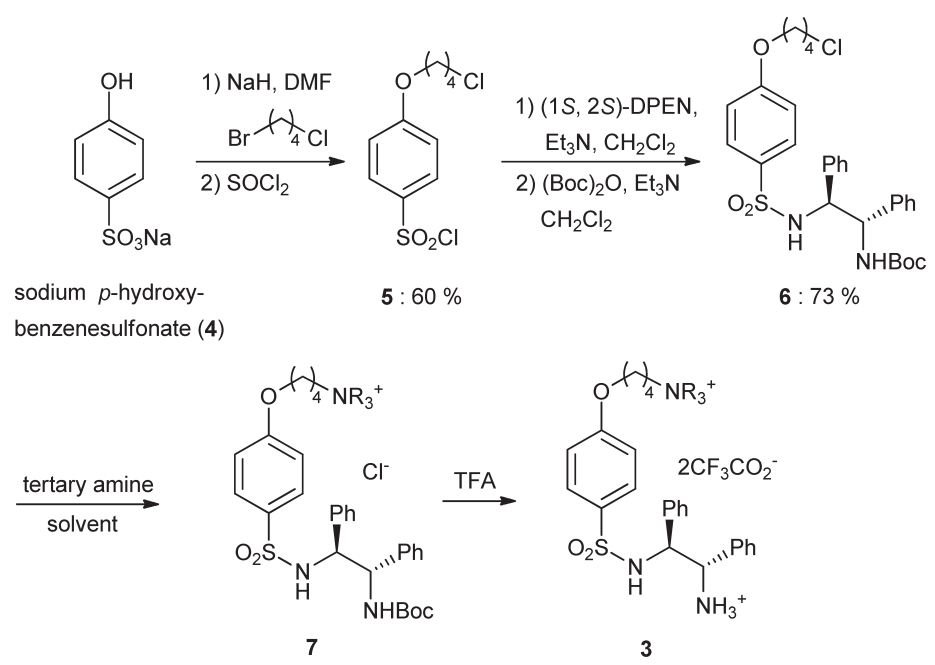

Chart 1. Synthesis of Chiral Ionic Ligand 3

of phenolic hydroxyl group and chlorination by using $\mathrm{SOCl}_{2}$ gave sulfonyl chloride 5. Introduction of $(1 S, 2 S)$-DPEN as an asymmetry source and followed by reaction of the resulting amine with di-tert-butoxycarbonate afforded carbamates $\mathbf{6}$ in $44 \%$ yield in four steps. Alkyl chloride 6 was allowed to react with a variety of amines, which formed the corresponding quaternary ammoniums 7 in relatively good to moderate yield (Table 1$)$. In the case of $N$-alkylation to quaternary ammonium $(6 \rightarrow 7)$, neat conditions were required to form $7 \mathbf{a}-\mathbf{c}$, e (entries 1-3,5), but in the case of 1-methylbenzimidazole and $N, N$-dimethyl-1-butanamine, $\mathrm{CH}_{3} \mathrm{CN}$ was used as a solvent (entries 4, 6). Finally, cleavage of the tert-butoxycarbonyl group of $\mathbf{7}$ by trifluoroacetic acid gave the desired ligand $\mathbf{3}$ in excellent yield. The structures of the synthesized ligand compounds were confirmed by IR and NMR $\left({ }^{1} \mathrm{H},{ }^{13} \mathrm{C},{ }^{19} \mathrm{~F}\right)$ spectra, MS, and elemental analysis.

The catalytic ability and recyclability of ligands $\mathbf{3 b}-\mathbf{f}$ were evaluated for RCATH of acetophenone, in comparison with the results for ligand $\mathbf{3 a}$ and catalyst $\mathbf{2}$ under the same conditions (Table 2). We confirmed good catalytic ability at the 1st cycle using ligands $\mathbf{3 b}-\mathbf{f}$ as well as ligand $\mathbf{3 a}$ and catalyst $\mathbf{2}$, whereas the recyclability of the IL phase including them was inferior to that of $\mathbf{3 a}$ after the 2 nd cycle. Although we had predicted that the cationic part of the ligand would be important for immobilization of the ruthenium complex in the IL phase in our previous report, there was more to good recyclability in the case of using 3a than just that. Surprisingly, even when 3b having a 2,3-dimethylimidazolium group was used as a ligand, the reaction efficiency after the 2 nd cycle resulted in a significant decrease. In addition, the signal believed to be that of the dihydropyridine part ${ }^{27)}$ was slightly observed in the ${ }^{1} \mathrm{H}-\mathrm{NMR}$ spectrum of the extract of the reaction using ligand 3c. Thus, the stability of the ligand may affect the maintenance of catalytic activity in RCATH.

We also synthesized ligand $\mathbf{1 4}$ and $\mathbf{1 5}$ of which the 1methylimidazolium group as a cationic moiety is connected to the TsDPEN ligand by a methylene group of different length from that of 3a, e.g., $n=2$ and 6 , respectively (Chart 2). For the synthesis of $\mathbf{1 4}$ bearing an ethylene group as a linker moiety, when 1-bromo-2-chloroethane was used as an alkylating 
Table 1. Preparation of Carbamates $7 \mathbf{a}-\mathbf{f}$ and Ligands $\mathbf{3 a}-\mathbf{f}$

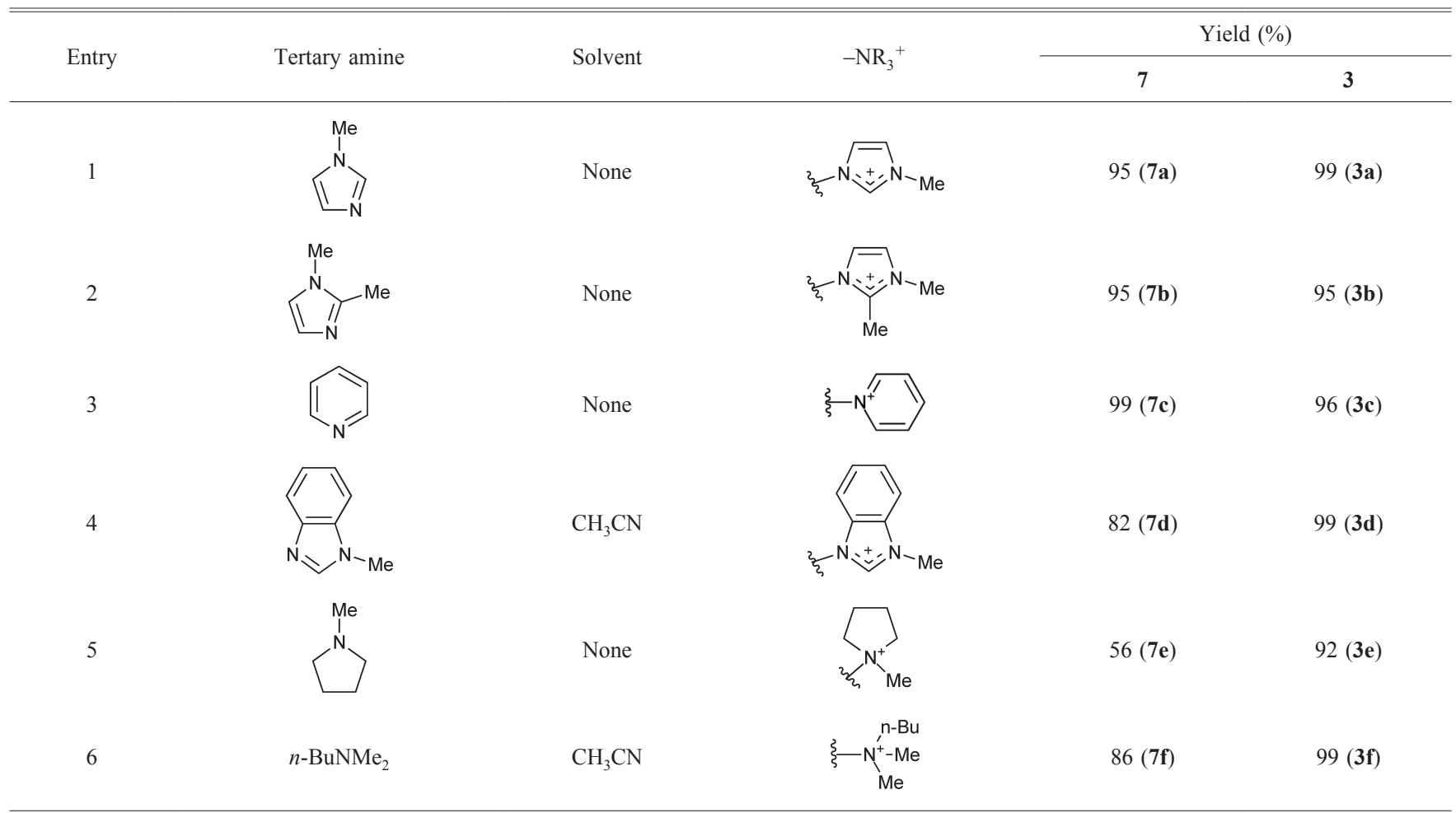

Table 2. RCATH of Acetophenone with Ligands $\mathbf{3 a}-\mathbf{f}^{a)}$

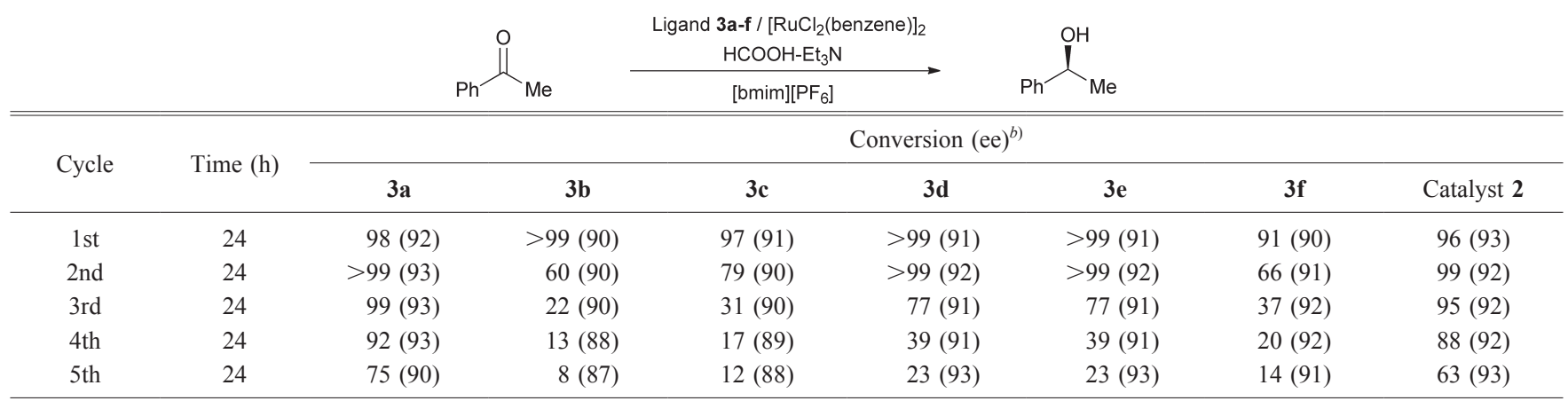

a) Reaction at room temperature for $24 \mathrm{~h}$ and $\mathrm{S} / \mathrm{C}=100 . b$ ) Determined by capillary GLC analysis using a chiral Cyclodex-B column.

reagent of sodium $p$-hydroxybenzenesulfonate similar to the synthesis of $\mathbf{3 a}$, sulfonyl chloride $\mathbf{8}$ was obtained in low yield and the purification was difficult. Using 2-(2-bromoethoxy)tetrahydro- $2 H$-pyran as an alternative to dihaloalkane gave $\mathbf{8}$ in moderate yield. Sulfonyl chloride $\mathbf{9}$ could be prepared by using dihaloalkane for preparation of $\mathbf{4}$. Chlorides 8 and 9 were converted to the desired ligands $\mathbf{1 4}$ and $\mathbf{1 5}$ by a method similar to synthesis of $\mathbf{3 a}$.

For comparison of ligands $\mathbf{1 4}$ and $\mathbf{1 5}$ with $\mathbf{3 a}$, RCATH of acetophenone was performed with $\mathbf{1 4}$ and $\mathbf{1 5}$ under the above conditions. As summarized in Table 3, using both $\mathbf{1 4}$ and $\mathbf{1 5}$ led to excellent conversion at the 1 st and 2 nd cycle, but with a sudden decrease after the 3rd cycle. In addition, enantioselectivity in the case of using them was slightly lower than when 3a was used. These results indicated that the tetramethylene group $\left(\mathrm{CH}_{2}\right)_{4}$ of $\mathbf{3 a}$ was suitable at a distance as a linker for our recycling reaction.

To confirm in more detail the effects of ligand structure on recyclability, we measured the residual quantity of ruthenium in the IL phase after the RCATH reaction. After the 5th cycle of the reaction using catalyst $\mathbf{1}$, the amounts of ligand $\mathbf{3 a}, \mathbf{b}$, 14 and $\mathbf{1 5}$ and the remaining ruthenium in the IL phase were measured by inductively coupled plasma (ICP)-MS. As shown in Table 4, a measurable amount of rutheniumremained in the IL phase containing ligand 3a (>99\%), which was the best result among the tested ligands. However, conversion decreased to $75 \%$ at the 5 th cycle when ligand 3 a was used. In the IL phase using 3 b having a 2,3-dimethylimidazolium group, the residual ruthenium decreased to $70.3 \%$, which is the same level as that of catalyst $2(68.3 \%)$. However, the conversion to 1-phenylethanol at the 5 th cycle with $\mathbf{3 b}$ and $\mathbf{2}$ were $8 \%$ and $63 \%$, respectively. These results indicated that a decrease of recyclability (or catalytic activity in IL) is not only caused by elution of $\mathrm{Ru}$ from the IL layer. As expected from the result of RCATH, a decrease in the residual quantity of ruthenium was observed in the IL phase used for 15 (29.7\%). This is thought to be due to the dissolving of the ruthenium complex in organic solvent during extraction of the product because 


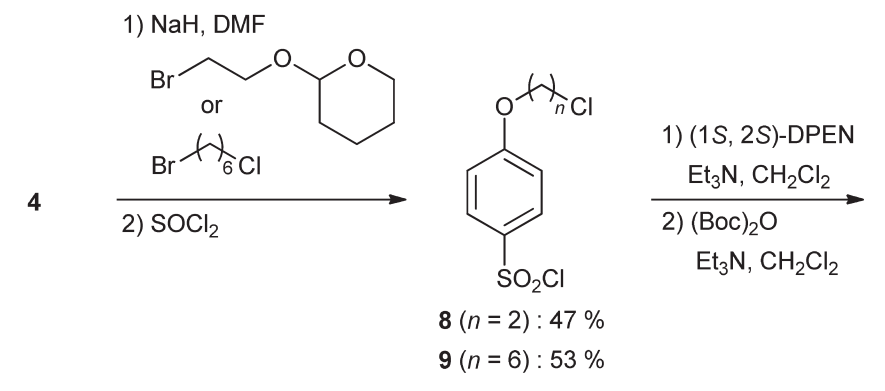

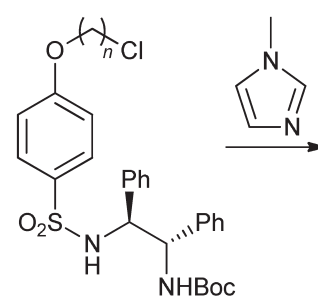

$10(n=2): 77 \%$

$11(n=6): 72 \%$<smiles>Cn1cccc1-c1ccccc1</smiles>

$12(n=2): 93 \%$

$13(n=6): 94 \%$

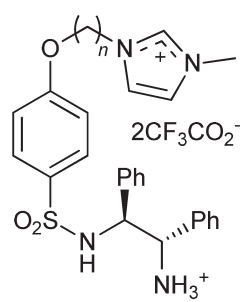

$14(n=2): 99 \%$

$15(n=6): 97 \%$

Chart 2. Preparation of Chiral Ionic Ligands $\mathbf{1 4}$ and $\mathbf{1 5}$

Table 3. RCATH of Acetophenone with Ligands 3a, 14 and $\mathbf{1 5}^{a}$

\begin{tabular}{ccrcr}
\hline \hline & & \multicolumn{3}{c}{ Conversion $(\mathrm{ee})^{b)}$} \\
\cline { 3 - 5 } Cycle & Time (h) & $\mathbf{1 4}(n=2)$ & 3a $(n=4)$ & $\mathbf{1 5}(n=6)$ \\
\hline 1st & 24 & $>99(90)$ & $98(92)$ & $>99(87)$ \\
2nd & 24 & $94(91)$ & $>99(93)$ & $98(91)$ \\
3rd & 24 & $44(90)$ & $99(93)$ & $75(91)$ \\
4 th & 24 & $22(90)$ & $92(93)$ & $37(90)$ \\
5 th & 24 & $14(89)$ & $75(90)$ & $22(84)$ \\
\hline
\end{tabular}

a) Reaction at room temperature for $24 \mathrm{~h}$ and $\mathrm{S} / \mathrm{C}=100 . b$ ) Determined by capillary GLC analysis using a chiral Cyclodex-B column.

Table 4. Percentages of Remaining Ru in IL after the 5th Cycle of RCATH

\begin{tabular}{|c|c|c|c|c|c|}
\hline & \multirow{2}{*}{ Catalyst 2} & \multicolumn{4}{|c|}{$\mathrm{Ru} /$ ligand complex } \\
\hline & & $3 \mathbf{a}$ & $\mathbf{3 b}$ & 14 & 15 \\
\hline$\%$ of remaining $\mathrm{Ru}$ in IL & 68.3 & $>99$ & 70.3 & 20.0 & 29.7 \\
\hline
\end{tabular}

the lipophilicity of $\mathbf{1 5}$ bearing a long alkyl chain seems to be higher than $\mathbf{3 a}$. While contrary to our expectation, the decrease was also observed in the IL phase used for 14 (20.0\%). This unexpected result may be due to a change in the structure of ligand $\mathbf{1 4}$ by elimination of the imidazolium group.

On the basis of these results, we hypothesized that lower solubility in organic solvent and ligand stability during the reaction are important factors for recyclability in this RCATH system.

We examined RCATH using different prochiral ketones as the substrate at every cycle with ligand 3a, which showed the best catalytic activity and recyclability among our ligands (Table 5). When acetophenone derivatives bearing an electronwithdrawing group were used as a substrate, the activity did not decrease even at the 3rd cycle (entry 3). On the other hand, when acetophenone derivative bearing an electron-donating group and propiophenone were used, the reaction was performed with a substrate/catalyst $(\mathrm{S} / \mathrm{C})$ mole ratio of 50 and a decrease of conversion was found at the 3 rd cycle (entry 6). The reaction with 3a using $\beta$-tetralone and cyclohexyl methyl ketone, which are generally known to be unsuitable ketones for asymmetric transfer hydrogenation, was examined (entries 7, 8). At the 1st cycle using cyclohexyl methyl ketone, the conversion was relatively good but enantioselectivity was very low. Next, using $\beta$-tetralone at the 2 nd cycle, conversion and enantioselectivity were moderate. These results of conversion and enantioselectivity decreasing when aliphatic and cyclic ketones were used agreed well with the results of transfer hydrogenation reported previously. ${ }^{4,28}$ It is noteworthy that in this RCATH system, both substrate and product did not influence the next reaction cycle.

\section{Conclusion}

Using TsDPEN ligand attached to a variety of ionic groups, an obvious difference was found in catalytic ability and recyclability. Furthermore, ICP-MS of the IL phase after the reaction suggested that consideration of both the residual quantity of ruthenium and the stability of the ruthenium complex with ligand should be required. Namely, the structure of 3a could make the main contribution to stabilization of the ruthenium complex in the IL phase, with the selection of suitable ionic groups being needed for high retention. Also, it was clearly shown that our ligand is useful for RCATH using a different ketone at every cycle. On the basis of these studies, further optimization of the ligand and application of the reaction to obtain bioactive compounds are currently underway in our laboratory.

\section{Experimental}

Melting points were measured with a Yanaco MP micromelting-point apparatus and are uncorrected. ${ }^{1} \mathrm{H}-,{ }^{13} \mathrm{C}$ - and ${ }^{19} \mathrm{~F}-\mathrm{NMR}$ spectra were measured on Varian INOVA $400 \mathrm{NB}$ $\left({ }^{1} \mathrm{H}: 400 \mathrm{MHz},{ }^{13} \mathrm{C}: 100 \mathrm{MHz},{ }^{19} \mathrm{~F}: 376 \mathrm{MHz}\right)$ and the chemical shifts are expressed in parts per million (ppm) downfield from tetramethylsilane as the internal standard $\left({ }^{19} \mathrm{~F}-\mathrm{NMR}\right.$ spectra 
Table 5. RCATH of Ketones with Ligand 3a

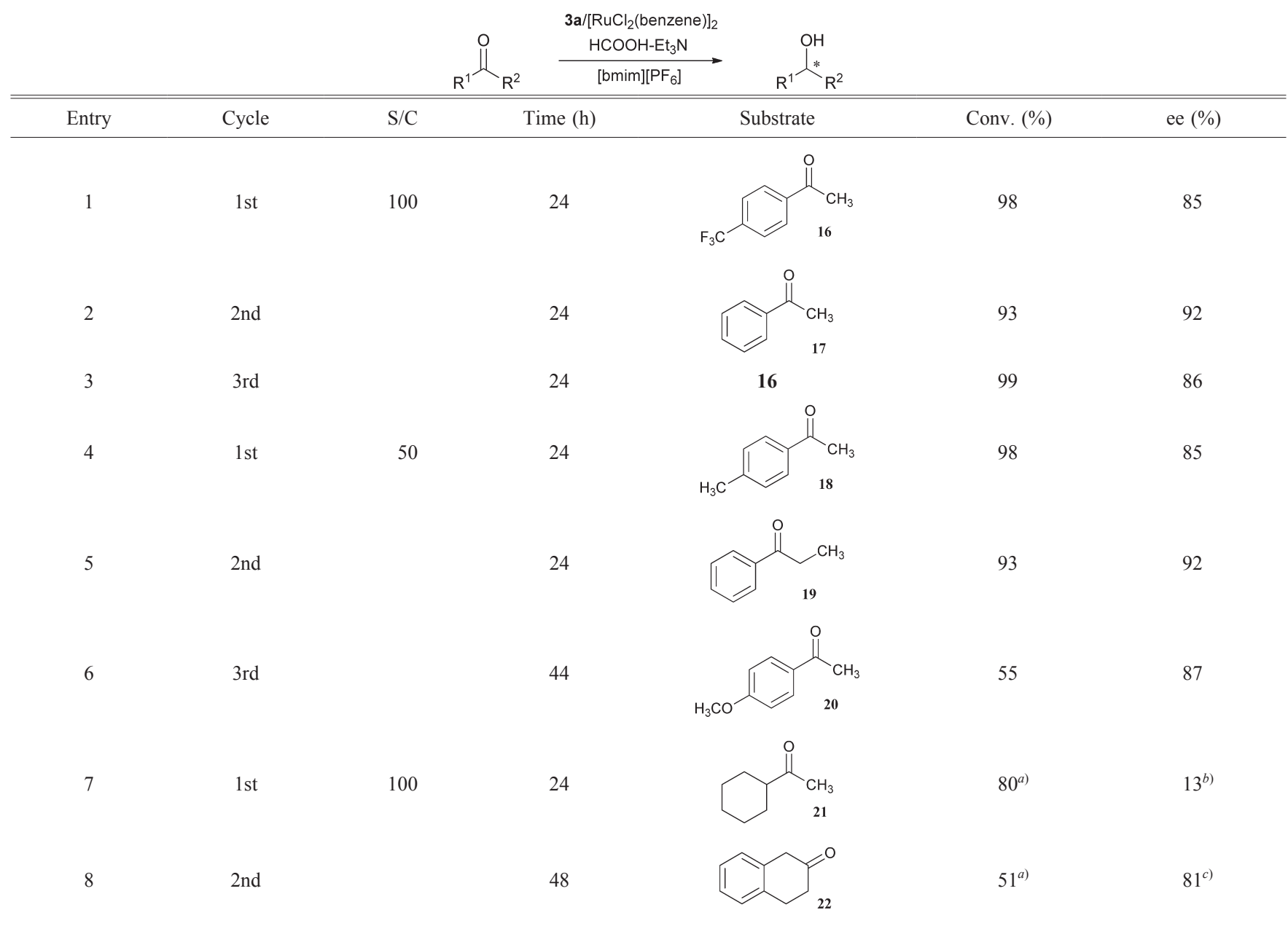

a) Determined by GLC. $b$ ) Determined by ${ }^{19}$ F-NMR after derived to Mosher's ester. ${ }^{29)} c$ ) Determined by HPLC.

were taken with trifluoroacetic acid as an external standard). Chemical shifts $(\delta)$ are given in ppm and $J$ values in Hz. Splitting patterns are designated as s, singlet; br s, broad singlet; $d$, doublet; dd, doublet of doublet; $t$, triplet; $m$, multiplet. Infrared (IR) spectra were recorded in the range $4000-600 \mathrm{~cm}^{-1}$ either as a $\mathrm{CHCl}_{3}$ solution technique for liquids or $\mathrm{KBr}$ pellets for solid samples. Mass spectra were measured on JEOL JMS SX-102 A (FAB) or JEOL-JMS-GC Mate (EI, CI) MS spectrometer. Elemental analyses were performed on a PerkinElmer, Inc. 2400 Series II CHNS/O Analyzer. Optical rotations were measured with a Horiba SEPA-200 in a $5-\mathrm{cm}$ cell. GLC was performed on a Shimadzu GC-15A equipped with a J\&W Scientific chiral Cyclodex-B column $(\phi 0.25 \mathrm{~mm} \times 30 \mathrm{~m})$. Helium was used as the carrier gas and FID as the detector. Shimadzu LC-6A was used as the pump, Shimadzu SPD-6A as the UV detector and Daicel Chemical Chiralpak (AD-H, $\phi 0.46 \mathrm{~cm} \times 25 \mathrm{~cm})$ for HPLC. Silica gel column chromatography was performed with Merck Kieselgel 60 (70-230 mesh). Alumina column chromatography was performed with Nacalai activated alumina (ca. 300 mesh). Preparative thin-layer chromatography was performed on Nacalai silica gel 60PF254 pre-coated plates. ICP-MS was measured with Shimadzu ICPM-8500.

4-(4-Chlorobutoxy)benzenesulfonyl Chloride (5) ${ }^{30)} \mathrm{NaH}$ (60\% in mineral oil, $80 \mathrm{mg}, 2.00 \mathrm{mmol}$ ) was added to a solu- tion of 4-hydroxybenzenesulfonic acid sodium salt dihydrate (464 mg, $2.00 \mathrm{mmol}$ ) in $N, N$-dimethylformamide (DMF) $(5.00 \mathrm{~mL})$ at $0^{\circ} \mathrm{C}$ under $\mathrm{N}_{2}$. After stirring for $1 \mathrm{~h}$ at $0^{\circ} \mathrm{C}$, 1-bromo-4-chlorobutane $(0.230 \mathrm{~mL}, 2.00 \mathrm{mmol})$ was added to the solution. The mixture was stirred for $12 \mathrm{~h}$ at $100^{\circ} \mathrm{C}$. The solvent was removed under reduced pressure and AcOEt $(15.0 \mathrm{~mL})$ was added to the residue. The resulting precipitate was collected by filtration. The precipitate was dried under reduced pressure. A mixture of the precipitate and $\mathrm{SOCl}_{2}$ $(7.0 \mathrm{~mL}, 80 \mathrm{mmol})$ was heated at $90^{\circ} \mathrm{C}$ for $13 \mathrm{~h}$. The reaction mixture was poured into ice water. After stirring for $5 \mathrm{~min}$, the product was extracted with $\mathrm{CHCl}_{3}(20 \mathrm{~mL} \times 3)$. The organic layer was dried over $\mathrm{Na}_{2} \mathrm{SO}_{4}$, filtered and evaporated. The crude product was purified by column chromatography (AcOEt: $n$-hexane $=1: 5$ ) to give compound 5. Yield: $338 \mathrm{mg}$ $(60 \%)$. Light yellow oil. ${ }^{1} \mathrm{H}-\mathrm{NMR}\left(\mathrm{CDCl}_{3}\right) \delta: 1.96-2.05(4 \mathrm{H}$, $\left.\mathrm{m}, 2 \times \mathrm{CH}_{2}\right), 3.63\left(2 \mathrm{H}, \mathrm{t}, J=6.2 \mathrm{~Hz}, \mathrm{CH}_{2} \mathrm{Cl}\right), 4.12(2 \mathrm{H}, \mathrm{t}$, $\left.J=5.9 \mathrm{~Hz}, \mathrm{CH}_{2} \mathrm{O}\right), 7.03\left(2 \mathrm{H}, \mathrm{d}, J=9.3 \mathrm{~Hz}, \overline{\mathrm{SO}}_{2} \mathrm{Ar}-\mathrm{H}\right), 7.97(2 \mathrm{H}$, $\left.\mathrm{d}, J=9.2 \mathrm{~Hz}, \mathrm{SO}_{2} \mathrm{Ar}-\underline{\mathrm{H}}\right) .{ }^{13} \mathrm{C}-\mathrm{NMR}\left(\mathrm{CDCl}_{3}\right) \delta: 26.3,29.0,44.4$, 67.9, 115.1, 129.5, 136.0, 164.2. IR $\left(\mathrm{CHCl}_{3}\right) \mathrm{cm}^{-1}: 2917,1588$, 1490, 1368, 1257, 1158, 573. High resolution (HR)-MS electron ionization (EI) $m / z$ : Found, 281.9883 (Calcd for $\mathrm{C}_{10} \mathrm{H}_{12} \mathrm{Cl}_{2} \mathrm{O}_{3} \mathrm{~S}$ : 281.9884). MS (EI) $m / z$ (rel. int. \%): 58 (100), 91 (70), 157 (18), 247 (9), $282\left(\mathrm{M}^{+}, 1.8\right), 284\left(\mathrm{M}^{+}+2,1.1\right), 286\left(\mathrm{M}^{+}+4,0.4\right)$.

4-(2-Chloroethoxy)benzenesulfonyl Chloride (8) ${ }^{30)}$ Com- 
pound 8 was synthesized from $4(1.16 \mathrm{~g}, 5.00 \mathrm{mmol})$ and 2-(2-bromoethoxy)-tetrahydro- $2 H$-pyran $(0.831 \mathrm{~mL}, 5.50 \mathrm{mmol})$ in the same manner as the synthesis of $\mathbf{5}$. Yield: $598 \mathrm{mg}(47 \%)$. Light yellow oil. ${ }^{1} \mathrm{H}-\mathrm{NMR}\left(\mathrm{CDCl}_{3}\right) \delta: 3.87(2 \mathrm{H}, \mathrm{t}, J=5.7 \mathrm{~Hz}$, $\left.\mathrm{CH}_{2} \mathrm{Cl}\right), 4.34\left(2 \mathrm{H}, \mathrm{t}, J=5.8 \mathrm{~Hz}, \mathrm{CH}_{2} \mathrm{O}\right), 7.08(2 \mathrm{H}, \mathrm{d}, J=9.0 \mathrm{~Hz}$, $\left.\mathrm{SO}_{2} \mathrm{Ar}-\mathrm{H}\right), \quad 8.00 \quad\left(2 \mathrm{H}, \quad \mathrm{d}, \quad J=9 . \overline{2 \mathrm{~Hz}}, \quad \mathrm{SO}_{2} \mathrm{Ar}-\underline{\mathrm{H}}\right) . \quad{ }^{13} \mathrm{C}-\mathrm{NMR}$ $\left(\mathrm{CDCl}_{3}\right) \delta: 41.3,68.5,115.2,129.6,136.8,163.4$. IR $\left(\mathrm{CHCl}_{3}\right)$ $\mathrm{cm}^{-1}:$ 3050, 2930, 1765, 1589, 1578, 1489, 1371, 1260, 1183, 1165, 574, 557. HR-MS (EI) $\mathrm{m} / \mathrm{z}$ : Found, 253.9571 (Calcd for $\mathrm{C}_{8} \mathrm{H}_{8} \mathrm{Cl}_{2} \mathrm{O}_{3} \mathrm{~S}:$ 253.9571). MS m/z (rel. int. \%): 63 (100), 92 (9), 155 (13), 219 (66), $254\left(\mathrm{M}^{+}, 12\right), 256\left(\mathrm{M}^{+}+2,8\right), 258\left(\mathrm{M}^{+}+4\right.$, 2).

4-(6-Chlorohexyloxy)benzenesulfonyl Chloride (9) ${ }^{30)}$ Compound 9 was synthesized from $4(464 \mathrm{mg}, 2.00 \mathrm{mmol})$ and 1-bromo-6-chlorohexane $(0.300 \mathrm{~mL}, 2.00 \mathrm{mmol})$ in the same manner as the synthesis of 5. Yield: $331 \mathrm{mg}$ (53\%). Light yellow oil. ${ }^{1} \mathrm{H}-\mathrm{NMR}\left(\mathrm{CDCl}_{3}\right) \delta: 1.48-1.58\left(4 \mathrm{H}, \mathrm{m}, 2 \times \mathrm{CH}_{2}\right)$, 1.79-1.89 (4H, m, $\left.2 \times \mathrm{CH}_{2}\right), 3.56\left(2 \mathrm{H}, \mathrm{t}, J=6.6 \mathrm{~Hz}, \mathrm{CH}_{2} \overline{\mathrm{Cl}}\right)$, $4.07\left(2 \mathrm{H}, \mathrm{t}, J=6.4 \mathrm{~Hz}, \widehat{\mathrm{CH}_{2} \mathrm{O}}\right), 7.03\left(2 \mathrm{H}, \mathrm{d}, J=9.0 \mathrm{~Hz}, \overrightarrow{\mathrm{SO}_{2} \mathrm{Ar}-}\right.$ $\mathrm{H}), 7.96\left(2 \mathrm{H}, \mathrm{d}, J=9.2 \mathrm{~Hz}, \mathrm{SO}_{2} \mathrm{Ar}-\mathrm{H}\right) .{ }^{13} \mathrm{C}-\mathrm{NMR}\left(\mathrm{CDCl}_{3}\right) \delta$ : 25.2, 26.5, 28.7, 32.4, 44.9, 68.6, 115.0, 129.5, 135.8, 164.4. IR $\left(\mathrm{CHCl}_{3}\right) \mathrm{cm}^{-1}:$ 2920, 2850, 1588, 1573, 1490, 1370, 1260, 1183, 1162, 575, 554. HR-MS (EI) $\mathrm{m} / \mathrm{z}$ : Found, 310.0203 (Calcd for $\mathrm{C}_{12} \mathrm{H}_{16} \mathrm{Cl}_{2} \mathrm{O}_{3} \mathrm{~S}$ : 310.0197). MS (EI) $\mathrm{m} / \mathrm{z}$ (rel. int. \%): 83 (100), 94 (12), 157 (22), 192 (10), 275 (13), $310\left(\mathrm{M}^{+}, 18\right), 312\left(\mathrm{M}^{+}+2\right.$, 12), $314\left(\mathrm{M}^{+}+4,3\right)$.

$N$-[(1S,2S)-2-[(1,1-Dimethylethoxy)carbonyl]amino-1,2diphenylethyl]-4-(4-chlorobutoxy)benzenesulfonamide (6) $(1 S, 2 S)-(-)-1,2-D P E N \quad(498 \mathrm{mg}, 2.34 \mathrm{mmol})$ and $\mathrm{Et}_{3} \mathrm{~N}$ $(0.340 \mathrm{~mL}, 2.44 \mathrm{mmol})$ was added to a solution of $5(346 \mathrm{mg}$, $1.22 \mathrm{mmol})$ in $\mathrm{CH}_{2} \mathrm{Cl}_{2}(3.00 \mathrm{~mL})$ at $0^{\circ} \mathrm{C}$ under $\mathrm{N}_{2}$. After stirring for $22 \mathrm{~h}$ at r.t., solution of (Boc) ${ }_{2} \mathrm{O}(400 \mathrm{mg}, 1.83 \mathrm{mmol})$ and $\mathrm{Et}_{3} \mathrm{~N}(0.255 \mathrm{~mL}, 1.83 \mathrm{mmol})$ in $\mathrm{CH}_{2} \mathrm{Cl}_{2}(2.00 \mathrm{~mL})$ was added to the solution under $\mathrm{N}_{2}$. The mixture was stirred for $24 \mathrm{~h}$ at r.t. The solvent was removed under reduced pressure and sat. $\mathrm{NaHCO}_{3}(5.0 \mathrm{~mL})$ was added to the residue. The product was extracted with AcOEt $(20 \mathrm{~mL} \times 3)$. The organic layer was dried over $\mathrm{Na}_{2} \mathrm{SO}_{4}$, filtered and evaporated. The crude product was purified by column chromatography (AcOEt: $n$ hexane $=1: 3$ ) to give the compound 6. Yield: $498 \mathrm{mg}(73 \%)$. White needles (recryst. from AcOEt). mp 196.5-197.0 ${ }^{\circ} \mathrm{C}$. $[\alpha]_{\mathrm{D}}^{27}-21.00\left(c=1.00, \mathrm{CHCl}_{3}\right) .{ }^{1} \mathrm{H}-\mathrm{NMR}\left(\mathrm{CDCl}_{3}\right) \delta: 1.47(9 \mathrm{H}$, $\mathrm{s}, t$-Bu), $1.90-2.00\left(4 \mathrm{H}, \mathrm{m}, 2 \times \mathrm{CH}_{2}\right), 3.61(2 \mathrm{H}, \mathrm{t}, J=6.2 \mathrm{~Hz}$, $\left.\mathrm{CH}_{2} \mathrm{Cl}\right), 3.95\left(2 \mathrm{H}, \mathrm{t}, J=5.6 \mathrm{~Hz}, \overline{\mathrm{CH}}_{2} \mathrm{O}\right), 4.56(1 \mathrm{H}, \mathrm{dd}, J=7.0$, $9.7 \mathrm{~Hz}, \mathrm{CH}(\mathrm{Ph}) \mathrm{NH}), 4.78(1 \mathrm{H}, \mathrm{t}, \bar{J}=9.5 \mathrm{~Hz}, \mathrm{CH}(\mathrm{Ph}) \mathrm{NH}), 5.25$ $(1 \mathrm{H}, \mathrm{d}, \bar{J}=8.1 \mathrm{~Hz}, \mathrm{NH}), 6.08(1 \mathrm{H}$, brs, NH), $6.67(2 \mathrm{H}, \mathrm{d}$, $\left.J=9.0 \mathrm{~Hz}, \mathrm{SO}_{2} \mathrm{Ar}-\underline{\mathrm{H}}\right), \overline{6} .77-7.17(10 \mathrm{H}, \mathrm{m}, 2 \times \overline{\mathrm{Ph}}-\underline{\mathrm{H}}), 7.46(2 \mathrm{H}$, d, $\left.J=9.0 \mathrm{~Hz}, \mathrm{SO}_{2} \mathrm{Ar}-\mathrm{H}\right) .{ }^{13} \mathrm{C}-\mathrm{NMR}\left(\mathrm{CDCl}_{3}\right) \delta: 26.4,28.3$, 29.1, 44.5, 60.0, 63.9, 67.2, 80.6, 114.1, 127.2, 127.3, 127.4, $127.9,128.0,128.2,128.5,129.0,133.1,137.8,138.1,161.6$. IR $\left(\mathrm{CHCl}_{3}\right) \mathrm{cm}^{-1}: 3410,3350,2950,1686,1593,1491,1152$. HR-MS (FAB+) $m / z$ : Found, $559.2039(\mathrm{M}+1)^{+}$(Calcd for $\mathrm{C}_{29} \mathrm{H}_{36} \mathrm{ClN}_{2} \mathrm{O}_{5} \mathrm{~S}:$ 559.2033). Anal. Calcd for $\mathrm{C}_{29} \mathrm{H}_{35} \mathrm{ClN}_{2} \mathrm{O}_{5} \mathrm{~S}: \mathrm{C}$, 62.30; H, 6.31; N, 5.01. Found: C, 62.54; H, 6.49; N, 5.01.

$N$-[(1S,2S)-2-[(1,1-Dimethylethoxy)carbonyl]amino-1,2diphenylethyl]-4-(2-chloroethoxy)benzenesulfonamide (10) Compound $\mathbf{1 0}$ was synthesized from $\mathbf{8}(593 \mathrm{mg}, 2.34 \mathrm{mmol})$ in the same manner as the synthesis of 6. Yield: $962 \mathrm{mg}(77 \%)$. White needles (recryst. from AcOEt). mp 206.6-207.1 ${ }^{\circ} \mathrm{C} .[\alpha]_{\mathrm{D}}^{25}$ $-23.24\left(c=0.80, \mathrm{CHCl}_{3}\right) .{ }^{1} \mathrm{H}-\mathrm{NMR}\left(\mathrm{CDCl}_{3}\right) \delta: 1.46(9 \mathrm{H}, \mathrm{s}$, $t$ - $\mathrm{Bu}), 3.78\left(2 \mathrm{H}, \mathrm{t}, J=5.8 \mathrm{~Hz}, \mathrm{CH}_{2} \mathrm{Cl}\right), 4.17(2 \mathrm{H}, \mathrm{t}, J=5.7 \mathrm{~Hz}$,
$\left.\mathrm{CH}_{2} \mathrm{O}\right), 4.58(1 \mathrm{H}, \mathrm{t}, J=8.3 \mathrm{~Hz}, \mathrm{CH}(\mathrm{Ph}) \mathrm{NH}), 4.80(1 \mathrm{H}, \mathrm{t}$, $J=8.8 \mathrm{~Hz}, \mathrm{CH}(\mathrm{Ph}) \mathrm{NH}), 5.31(1 \mathrm{H}, \overline{\mathrm{brs}}, \mathrm{NH}), 6.25(1 \mathrm{H}$, brs, $\mathrm{NH}), 6.68\left(\overline{2 \mathrm{H}}, \mathrm{d}, J=8.8 \mathrm{~Hz}, \mathrm{SO}_{2} \mathrm{Ar}-\underline{\mathrm{H}}\right), \overline{6} .77-7.16(10 \mathrm{H}, \mathrm{m}$, $2 \times \mathrm{Ph}-\underline{\mathrm{H}}), 7.46\left(2 \mathrm{H}, \mathrm{d}, J=8.8 \mathrm{~Hz}, \mathrm{SO}_{2} \mathrm{Ar}-\underline{\mathrm{H}}\right) .{ }^{13} \mathrm{C}-\mathrm{NMR}\left(\mathrm{CDCl}_{3}\right)$ $\delta: 28.3,41.5,60.0,64.0,68.1,80.7,114.2,127.3,127.38,127.44$, $127.9,128.0,128.5,129.0,133.3,137.7,138.0,156.9,160.8$. IR $\left(\mathrm{CHCl}_{3}\right) \mathrm{cm}^{-1}: 3410,3390,2998,2992,1687,1593,1580$, 1490, 1249, 1222, 1153. HR-MS (FAB+) $\mathrm{m} / z$ : Found, 553.1547 $(\mathrm{M}+\mathrm{Na})^{+} \quad$ (Calcd for $\mathrm{C}_{27} \mathrm{H}_{31} \mathrm{ClN}_{2} \mathrm{O}_{5} \mathrm{~S} \cdot \mathrm{Na}$ : 553.1540). Anal. Calcd for $\mathrm{C}_{27} \mathrm{H}_{31} \mathrm{ClN}_{2} \mathrm{O}_{5} \mathrm{~S}: \mathrm{C}, 61.06 ; \mathrm{H}, 5.88 ; \mathrm{N}, 5.27$. Found: $\mathrm{C}$, 60.82; H, 5.99; N, 5.03.

$N$-[(1S,2S)-2-[(1,1-Dimethylethoxy)carbonyl]amino-1,2diphenylethyl]-4-(6-chlorohexyloxy)benzenesulfonamide (11) Compound $\mathbf{1 1}$ was synthesized from 9 (164 mg, $0.527 \mathrm{mmol}$ ) in the same manner as the synthesis of $\mathbf{6}$. Yield: $222 \mathrm{mg}$ (72\%). White needles (recryst. from AcOEt). mp $183.1-183.8^{\circ} \mathrm{C} .[\alpha]_{\mathrm{D}}^{22}-21.38\left(c=0.72, \mathrm{CHCl}_{3}\right) .{ }^{1} \mathrm{H}-\mathrm{NMR}$ $\left(\mathrm{CDCl}_{3}\right) \delta: 1.47-1.52\left(13 \mathrm{H}, \mathrm{m}, t-\mathrm{Bu}\right.$ and $\left.2 \times \mathrm{CH}_{2}\right), 1.75-1.85$ $\left(4 \mathrm{H}, \mathrm{m}, 2 \times \mathrm{CH}_{2}\right), 3.55\left(2 \mathrm{H}, \mathrm{t}, J=6.6 \mathrm{~Hz}, \mathrm{CH}_{2} \mathrm{Cl}\right), 3.92(2 \mathrm{H}, \mathrm{t}$, $\left.J=6.4 \mathrm{~Hz}, \mathrm{CH}_{2} \mathrm{O}\right), 4.55(1 \mathrm{H}, \mathrm{dd}, J=7.0,9.9 \mathrm{~Hz}, \mathrm{CH}(\mathrm{Ph}) \mathrm{NH})$, $4.78(1 \mathrm{H}, \mathrm{t}, J=8.9 \mathrm{~Hz}, \mathrm{CH}(\mathrm{Ph}) \mathrm{NH}), 5.22(1 \mathrm{H}$, brd, $J=4.8 \mathrm{~Hz}$, $\mathrm{NH}), 6.03(1 \mathrm{H}$, brs, $\underline{\mathrm{H}}), 6.68\left(2 \mathrm{H}, \mathrm{d}, J=9.0 \mathrm{~Hz}, \mathrm{SO}_{2} \mathrm{Ar}-\underline{\mathrm{H}}\right)$, 6.76-7.17 (10H, m, 2×Ph- $\underline{\mathrm{H}}), 7.46\left(2 \mathrm{H}, \mathrm{d}, J=9.0 \mathrm{~Hz}, \mathrm{SO}_{2} \mathrm{Ar}-\underline{\mathrm{H}}\right)$. ${ }^{13} \mathrm{C}-\mathrm{NMR}\left(\mathrm{CDCl}_{3}\right) \delta: 25.3,26.5,28.3,28.8,32.4,44.9,60.0$, $63.9,68.0,80.6,114.1,127.3,127.4,127.5,127.9,128.0,128.5$, $128.9,132.3,137.8,138.1,156.8,161.8$. IR $\left(\mathrm{CHCl}_{3}\right) \mathrm{cm}^{-1}$ : 3360, 3300, 2920, 1680, 1590, 1512, 1330, 1256, 1150, 690, 570. HR-MS (FAB+) $m / z$ : Found, $587.2339(\mathrm{M}+1)^{+}($Calcd for $\mathrm{C}_{31} \mathrm{H}_{40} \mathrm{ClN}_{2} \mathrm{O}_{5} \mathrm{~S}:$ 587.2346). Anal. Calcd for $\mathrm{C}_{31} \mathrm{H}_{39} \mathrm{ClN}_{2} \mathrm{O}_{5} \mathrm{~S}: \mathrm{C}$, 63.41; H, 6.69; N, 4.77. Found: C, 63.69; H, 6.84; N, 4.81.

1-[4-[4-[[[(1S,2S)-2-[[(1,1-Dimethylethoxy)carbonyl]amino]-1,2-diphenylethyl]amino]sulfonyl]phenoxy]butyl]3-methyl-1H-imidazolium Chloride (7a) A mixture of $6(117 \mathrm{mg}, 0.209 \mathrm{mmol})$ and 1-methylimidazole $(0.167 \mathrm{~mL}$, $2.09 \mathrm{mmol}$ ) was heated at $88^{\circ} \mathrm{C}$ for $11 \mathrm{~h}$ under $\mathrm{N}_{2}$. The volatile material was removed under reduced pressure with a glass tube oven, and the residue was washed with AcOEt $(0.5 \mathrm{~mL} \times 10)$ to obtain compound 7a. Yield: $128 \mathrm{mg}(95 \%)$. Yellow oil. $[\alpha]_{\mathrm{D}}^{24}-33.07\left(c=1.30, \mathrm{CH}_{3} \mathrm{OH}\right) .{ }^{1} \mathrm{H}-\mathrm{NMR}\left(\mathrm{CD}_{3} \mathrm{OD}\right)$ $\delta: 1.36(9 \mathrm{H}, \mathrm{s}, t-\mathrm{Bu}), 1.73-1.80\left(2 \mathrm{H}, \mathrm{m}, \mathrm{CH}_{2}\right), 1.98-2.05$ $\left(2 \mathrm{H}, \mathrm{m}, \mathrm{CH}_{2}\right), 3.87(3 \mathrm{H}, \mathrm{s}, \mathrm{N}-\mathrm{Me}), 3.94(2 \mathrm{H}, \mathrm{t}, J=6.2 \mathrm{~Hz}$, $\left.\mathrm{CH}_{2} \mathrm{O}\right), 4.25\left(2 \mathrm{H}, \mathrm{t}, J=7.3 \mathrm{~Hz}, \overline{\mathrm{CH}}_{2} \mathrm{~N}\right), 4.56(1 \mathrm{H}, \mathrm{d}, J=8.4 \mathrm{~Hz}$, C $\underline{H}(\mathrm{Ph}) \mathrm{NH}), 4.78(1 \mathrm{H}$, brd, $J=8.6 \mathrm{~Hz}, \mathrm{CH}(\mathrm{Ph}) \mathrm{NH}), 6.68(2 \mathrm{H}$, d, $\left.J=8.8 \mathrm{~Hz}, \quad \mathrm{SO}_{2} \mathrm{Ar}-\underline{\mathrm{H}}\right), 6.91-7.12(10 \mathrm{H}, \mathrm{m}, 2 \times \mathrm{Ph}-\underline{\mathrm{H}}), 7.37$ $\left(2 \mathrm{H}, \mathrm{d}, J=9.0 \mathrm{~Hz}, \mathrm{SO}_{2} \overline{\mathrm{A}} \mathrm{r}-\underline{\mathrm{H}}\right), 7.52(1 \mathrm{H}, \mathrm{d}, J=1.8 \mathrm{~Hz}$, imidazole$\underline{\mathrm{H}}), 7.61(1 \mathrm{H}, \mathrm{d}, J=1.8 \mathrm{~Hz}$, imidazole- $\underline{\mathrm{H}}), 8.94$ (1H, s, 2-imidazole-H). ${ }^{13} \mathrm{C}-\mathrm{NMR}$ (CD $\left.\mathrm{OD}\right) \delta$ : 26.7, 27.9, 28.7, 36.5, 50.4, 61.0, $64.0,68.5,80.5,115.3,123.5,124.9,128.1,128.2,128.3,128.6$, $129.0,129.1,129.8,130.0,134.0,139.8,140.9,157.7,162.9$. IR (KBr) $\mathrm{cm}^{-1}:$ 3340, 3214, 3041, 2919, 1696, 1592, 1511, 1318, 1248, 1150. HR-MS (FAB + ) $m / z$ : Found, $605.2803(\mathrm{M})^{+}$ (Calcd for $\mathrm{C}_{33} \mathrm{H}_{41} \mathrm{~N}_{4} \mathrm{O}_{5} \mathrm{~S}^{+}:$605.2798).

1-[4-[4-[[](1S,2S)-2-[[(1,1-Dimethylethoxy)carbonyl]amino]-1,2-diphenylethyl] amino]sulfonyl]phenoxy]butyl]-2,3-dimethyl-1H-imidazolium Chloride (7b) Compound $\mathbf{7 b}$ was synthesized from $\mathbf{6}(94 \mathrm{mg}, 0.168 \mathrm{mmol})$ in the same manner as the synthesis of $7 \mathbf{a}$. Yield: $105 \mathrm{mg}(95 \%)$. Yellow oil. $[\alpha]_{\mathrm{D}}^{22}-32.16\left(c=1.20, \mathrm{CH}_{3} \mathrm{OH}\right) .{ }^{1} \mathrm{H}-\mathrm{NMR}\left(\mathrm{CD}_{3} \mathrm{OD}\right)$ $\delta$ : $1.36(9 \mathrm{H}, \mathrm{s}, t-\mathrm{Bu}), 1.76-1.82\left(2 \mathrm{H}, \mathrm{m}, \mathrm{CH}_{2}\right), 1.91-1.98(2 \mathrm{H}$, $\left.\mathrm{m}, \mathrm{CH}_{2}\right), 2.59$ (3H, s, 2-imidazole-Me), 3.76 (3H, s, N-Me),

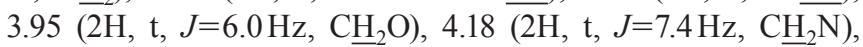


$4.56(1 \mathrm{H}, \mathrm{d}, J=8.1 \mathrm{~Hz}, \mathrm{CH}(\mathrm{Ph}) \mathrm{NH}), 4.77(1 \mathrm{H}, \mathrm{d}, J=7.7 \mathrm{~Hz}$, $\mathrm{CH}(\mathrm{Ph}) \mathrm{NH}), 6.68\left(2 \mathrm{H}, \mathrm{d}, J=9.0 \mathrm{~Hz}, \mathrm{SO}_{2} \mathrm{Ar}-\mathrm{H}\right), 6.88-7.12(10 \mathrm{H}$, $\mathrm{m}, 2 \times \mathrm{Ph}-\underline{\mathrm{H}}), 7.36\left(2 \mathrm{H}, \mathrm{d}, J=8.8 \mathrm{~Hz}, \mathrm{SO}_{2} \overline{\mathrm{Ar}}-\underline{\mathrm{H}}\right), 7.43(1 \mathrm{H}, \mathrm{d}$, $J=1.8 \mathrm{~Hz}$, imidazole-H), $7.50(1 \mathrm{H}, \mathrm{d}, J=1.8 \mathrm{~Hz}$, imidazole- $\underline{\mathrm{H}})$. ${ }^{13} \mathrm{C}-\mathrm{NMR}\left(\mathrm{CD}_{3} \mathrm{OD}\right) \bar{\delta}$ : 9.6, 26.8, 27.5, 28.7, 35.5, 49.1, 61.0, 64.1, 68.3, 80.5, 115.3, 122.1, 123.6, 128.1, 128.2, 128.3, 128.6, $129.0,129.1,129.8,130.1,134.0,139.9,145.8,157.7,162.9$. IR (KBr) $\mathrm{cm}^{-1}:$ 3367, 3218, 3125, 3040, 2911, 1697, 1591, 1251, 1148. HR-MS (FAB+) $m / z$ : Found, $619.2949(\mathrm{M})^{+}$(Calcd for $\mathrm{C}_{34} \mathrm{H}_{43} \mathrm{~N}_{4} \mathrm{O}_{5} \mathrm{~S}^{+}$: 619.2954).

1-[4-[4-[I[(1S,2S)-2-[[(1,1-Dimethylethoxy)carbonyl]amino]-1,2-diphenylethyl]amino]sulfonyl]phenoxy]butyl]pyridinium Chloride (7c) Compound 7c was synthesized from $6(145 \mathrm{mg}, 0.259 \mathrm{mmol})$ in the same manner as the synthesis of 7a. Yield: $164 \mathrm{mg}$ (99\%). Light brown crystals (washed with AcOEt). mp $169.5-170.4^{\circ} \mathrm{C}$. $[\alpha]_{\mathrm{D}}^{18}-30.58(c=1.00$, $\left.\mathrm{CH}_{3} \mathrm{OH}\right) .{ }^{1} \mathrm{H}-\mathrm{NMR}\left(\mathrm{CD}_{3} \mathrm{OD}\right) \delta: 1.36(9 \mathrm{H}, \mathrm{s}, t-\mathrm{Bu}), 1.77-1.84$ $\left(2 \mathrm{H}, \mathrm{m}, \mathrm{CH}_{2}\right), 2.11-2.18\left(2 \mathrm{H}, \mathrm{m}, \mathrm{CH}_{2}\right), 3.95(2 \mathrm{H}, \mathrm{t}, J=6.1 \mathrm{~Hz}$, $\left.\mathrm{CH}_{2} \mathrm{O}\right), 4 . \overline{57}(1 \mathrm{H}, \mathrm{d}, J=7.9 \mathrm{~Hz}, \overline{\mathrm{CH}}(\mathrm{Ph}) \mathrm{NH}), 4.68(2 \mathrm{H}, \mathrm{t}$, $\left.J=7.5 \mathrm{~Hz}, \mathrm{CH}_{2} \mathrm{~N}\right), 4.92(1 \mathrm{H}, \quad$ brd, $J=4.0 \mathrm{~Hz}, \mathrm{CH}(\mathrm{Ph}) \mathrm{NH})$, $6.68\left(2 \mathrm{H}, \mathrm{d}, J=9.0 \mathrm{~Hz}, \mathrm{SO}_{2} \mathrm{Ar}-\mathrm{H}\right), 6.93-7.17(10 \mathrm{H}, \mathrm{m}, 2 \times \mathrm{Ph}-$ $\underline{\mathrm{H}}), 7.37\left(2 \mathrm{H}, \mathrm{d}, J=9.0 \mathrm{~Hz}, \mathrm{SO}_{2} \mathrm{Ar}-\underline{\mathrm{H}}\right), 8.05(2 \mathrm{H}, \mathrm{t}, J=7.1 \mathrm{~Hz}$, 3,5-pyridine- $-\underline{H}), 8.52(1 \mathrm{H}, \mathrm{t}, J=7.9 \mathrm{~Hz}, 4$-pyridine- $\underline{\mathrm{H}}), 8.99$ $\left(2 \mathrm{H}, \mathrm{d}, J=5.7 \mathrm{~Hz}, 2,6\right.$-pyridine-H). ${ }^{13} \mathrm{C}-\mathrm{NMR}\left(\mathrm{CD}_{3} \mathrm{OD}\right) \delta: 26.6$, 28.7, 29.2, 61.0, 62.6, 64.0, 68.5, 80.3, 80.5, 115.3, 128.1, 128.2, $128.25,128.32,128.6,128.9,129.09,129.14,129.5,129.8$, $134.0,139.8,140.9,141.4,145.8,146.8,157.7,157.9,162.8(2$ sets of rotamer peaks appeared). IR $(\mathrm{KBr}) \mathrm{cm}^{-1}: 3340,3030$, 2950, 1674, 1630, 1590, 1489, 1404, 1360, 1250, 1148. HR-MS $(\mathrm{FAB}+) \mathrm{m} / \mathrm{z}$ : Found, $602.2693(\mathrm{M})^{+}$(Calcd for $\mathrm{C}_{34} \mathrm{H}_{40} \mathrm{~N}_{3} \mathrm{O}_{5} \mathrm{~S}^{+}$: 602.2689). Anal. Calcd for $\mathrm{C}_{34} \mathrm{H}_{40} \mathrm{ClN}_{3} \mathrm{O}_{5} \mathrm{~S}$ : C, 63.99; H, 6.32; N, 6.58. Found: C, 63.89; H, 6.51; N, 6.34.

1-[4-[4-[[[(1S,2S)-2-[[(1,1-Dimethylethoxy)carbonyl]amino]-1,2-diphenylethyl] amino]sulfonyl]phenoxy]butyl]3-methylbenzimidazolium Chloride (7d) 1-Methylbenzimidazole $(264 \mathrm{mg}, 2.00 \mathrm{mmol})$ was added to a solution of 6 $(112 \mathrm{mg}, 0.200 \mathrm{mmol})$ in $\mathrm{CH}_{3} \mathrm{CN}(1.00 \mathrm{~mL})$ at $0^{\circ} \mathrm{C}$ under $\mathrm{N}_{2}$. After stirring for $76.5 \mathrm{~h}$ at $80^{\circ} \mathrm{C}$, the solvent was removed under reduced pressure and the residue was purified by alumina column chromatography (AcOEt, $\mathrm{MeOH}$ ) to give compound 7d. Yield: $113 \mathrm{mg}$ (82\%). Yellow oil. $[\alpha]_{\mathrm{D}}^{25}-26.19$ $\left(c=1.00, \mathrm{CH}_{3} \mathrm{OH}\right) .{ }^{1} \mathrm{H}-\mathrm{NMR}\left(\mathrm{CD}_{3} \mathrm{OD}\right) \delta: 1.38(9 \mathrm{H}, \mathrm{s}, t-\mathrm{Bu})$, 1.86-1.91 (2H, m, $\left.\mathrm{CH}_{2}\right), 2.14-2.21\left(2 \mathrm{H}, \mathrm{m}, \mathrm{CH}_{2}\right), 3.99(3 \mathrm{H}, \mathrm{t}$, $\left.J=6.0 \mathrm{~Hz}, \underline{\mathrm{CH}}_{2} \mathrm{O}\right), 4.11(3 \mathrm{H}, \mathrm{s}, \mathrm{N}-\underline{\mathrm{Me}}), 4.52(\overline{\mathrm{H}}, \mathrm{d}, J=8.4 \mathrm{~Hz}$, $\mathrm{CH}(\mathrm{Ph}) \mathrm{NH}), 4.59\left(2 \mathrm{H}, \mathrm{t}, J=7.3 \overline{\mathrm{Hz}}, \mathrm{CH}_{2} \mathrm{~N}\right), 4.77(1 \mathrm{H}$, brd, $J=6.4 \mathrm{~Hz}, \quad \mathrm{CH}(\mathrm{Ph}) \mathrm{NH}), 6.65\left(2 \mathrm{H}, \mathrm{d}, \bar{J}=9.0 \mathrm{~Hz}, \mathrm{SO}_{2} \mathrm{Ar}-\underline{\mathrm{H}}\right)$,

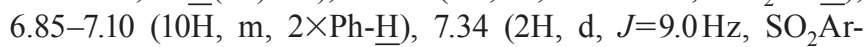
$\underline{\mathrm{H}}), 7.70-7.73(2 \mathrm{H}, \mathrm{m}$, benzimidazole- $\underline{\mathrm{H}}), 7.92-8.00(2 \mathrm{H}, \mathrm{m}$, benzimidazole- $\mathrm{H}), 8.53$ (1H, s, 2-benzimidazole- $-\mathrm{H}) .{ }^{13} \mathrm{C}-\mathrm{NMR}$ $\left(\mathrm{CDCl}_{3}\right) \delta: 25.9,26.0,28.3,33.4,47.0,59.6,63.4,67.0,79.5$, $112.6,112.8,113.8,126.5,126.88,126.93,127.1,127.2$, $127.6,127.8,128.2,128.7,128.8,131.0,132.0,133.1,138.4$, 144.4, 156.4, 160.9. IR (KBr) $\mathrm{cm}^{-1}: 3356,3217,3034,2924$, 2870, 1696, 1592, 1567, 1491, 1316, 1249, 1149, 697. HR-MS $(\mathrm{FAB}+) \mathrm{m} / \mathrm{z}$ : Found, $655.2960(\mathrm{M})^{+}\left(\right.$Calcd for $\mathrm{C}_{37} \mathrm{H}_{43} \mathrm{~N}_{4} \mathrm{O}_{5} \mathrm{~S}^{+}$: 655.2954).

1-[4-[4-[[](1S,2S)-2-[[(1,1-Dimethylethoxy)carbonyl]amino]-1,2-diphenylethyl]amino]sulfonyl]phenoxy]butyl]1-methylpyrrolidinium Chloride (7e) A mixture of 6 $(112 \mathrm{mg}, \quad 0.200 \mathrm{mmol})$ and 1-methylpyrrolidine $(0.208 \mathrm{~mL}$, $2.00 \mathrm{mmol}$ ) was heated at $80^{\circ} \mathrm{C}$ for $17 \mathrm{~h}$ under $\mathrm{N}_{2}$. The vola- tile material was removed under reduced pressure, and the residue was purified by alumina column chromatography $\left(\mathrm{CHCl}_{3}: \mathrm{MeOH}=5: 1\right)$ to obtain compound 7e. Yield: $72 \mathrm{mg}$ (56\%). Yellow amorphous. $[\alpha]_{\mathrm{D}}^{25}-26.39\left(c=1.00, \mathrm{CH}_{3} \mathrm{OH}\right)$. ${ }^{1} \mathrm{H}-\mathrm{NMR}\left(\mathrm{CD}_{3} \mathrm{OD}\right) \delta$ : $1.38(9 \mathrm{H}, \mathrm{s}, t-\mathrm{Bu}), 1.80-1.86(2 \mathrm{H}, \mathrm{m}$, $\left.\mathrm{CH}_{2}\right), 1.92-2.00\left(2 \mathrm{H}, \mathrm{m}, \mathrm{CH}_{2}\right), 2.21$ (4H, brs, 3,4-pyrrolidine$\underline{\mathrm{H}}), 3.06(3 \mathrm{H}, \mathrm{s}, \mathrm{N}-\mathrm{Me}), 3.42\left(2 \mathrm{H}\right.$, brt, $\left.J=8.4 \mathrm{~Hz}, \mathrm{CH}_{2} \mathrm{~N}\right)$, 3.49-3.56 (4H, m, 2,5-pyrrolidine- $\underline{\mathrm{H}}), 4.01(2 \mathrm{H}, \mathrm{t}, J=6.1 \mathrm{~Hz}$, $\left.\mathrm{CH}_{2} \mathrm{O}\right), 4.54(1 \mathrm{H}, \mathrm{d}, J=8.4 \mathrm{~Hz}, \overline{\mathrm{C}} \underline{\mathrm{H}}(\mathrm{Ph}) \mathrm{NH}), 4.78(1 \mathrm{H}, \mathrm{d}$, $J=5.1 \mathrm{~Hz}, \quad \underline{\mathrm{H}}(\mathrm{Ph}) \mathrm{NH}), 6.72\left(2 \mathrm{H}, \overline{\mathrm{d}}, J=9.0 \mathrm{~Hz}, \mathrm{SO}_{2} \mathrm{Ar}-\underline{\mathrm{H}}\right)$, 6.88-7.12 (10ㄱ, m, 2×Ph- $\underline{\mathrm{H}}), 7.38\left(2 \mathrm{H}, \mathrm{d}, J=9.0 \mathrm{~Hz}, \mathrm{SO}_{2} \overline{\mathrm{Ar}}-\right.$ $\underline{\mathrm{H}}) .{ }^{13} \mathrm{C}-\mathrm{NMR}\left(\mathrm{CD}_{3} \mathrm{OD}\right) \delta: 21.7,22.6,27.1,28.7,61.0,64.1$, $65.1,65.4,68.4,80.5,115.3,128.17,128.22,128.4,128.6$, $129.0,129.1,129.9,134.2,139.9,140.9,157.8,162.9$ (one aliphatic peak could not be found). IR (KBr) $\mathrm{cm}^{-1}$ : 3343, 3207, 3037, 3010, 2970, 2878, 1686, 1593, 1508, 1490, 1250, 1150, 696. HR-MS (FAB+) $\mathrm{m} / \mathrm{z}$ : Found, $608.3161(\mathrm{M})^{+}$(Calcd for $\left.\mathrm{C}_{34} \mathrm{H}_{46} \mathrm{~N}_{3} \mathrm{O}_{5} \mathrm{~S}^{+}: 608.3158\right)$.

1-[4-[4-[[](1S,2S)-2-[[(1,1-Dimethylethoxy)carbonyl]amino]-1,2-diphenylethyl] amino]sulfonyl]phenoxy]butyl]1-N-butyl- $\mathrm{N}, \mathrm{N}$-dimethylammonium Chloride (7f) Compound $7 \mathbf{f}$ was synthesized from $\mathbf{6}(112 \mathrm{mg}, 0.200 \mathrm{mmol})$ in the same manner as the synthesis of 7d. Yield: $114 \mathrm{mg}(86 \%)$. Colorless oil. $[\alpha]_{\mathrm{D}}^{25}-26.94 \quad\left(c=0.72, \mathrm{CH}_{3} \mathrm{OH}\right) .{ }^{1} \mathrm{H}-\mathrm{NMR}$ $\left(\mathrm{CD}_{3} \mathrm{OD}\right) \delta: 1.00\left(3 \mathrm{H}, \mathrm{t}, J=7.3 \mathrm{~Hz}, \mathrm{CH}_{3}\right), 1.34-1.45(11 \mathrm{H}$, $\mathrm{m}, t-\mathrm{Bu}$ and $\left.\mathrm{CH}_{2}\right), 1.69-1.77\left(2 \mathrm{H}, \mathrm{m}, \mathrm{CH}_{2}\right), 1.79-1.86(2 \mathrm{H}$, $\left.\mathrm{m}, \mathrm{CH}_{2}\right), 1.89-1.97\left(2 \mathrm{H}, \mathrm{m}, \mathrm{CH}_{2}\right), 3.07(6 \mathrm{H}, \mathrm{s}, 2 \times \mathrm{N}-\underline{\mathrm{Me}})$, $3.30\left(2 \mathrm{H}, \mathrm{t}, J=8.6 \mathrm{~Hz}, \mathrm{CH}_{2} \mathrm{~N}\right), 3.37(2 \mathrm{H}, \quad$ brt,$J=8.4 \mathrm{~Hz}$, $\left.\mathrm{CH}_{2} \mathrm{~N}\right), 4.02\left(2 \mathrm{H}, \mathrm{t}, J=6.0 \mathrm{~Hz}, \mathrm{CH}_{2} \mathrm{O}\right), 4.53(1 \mathrm{H}, \mathrm{d}, J=8.4 \mathrm{~Hz}$, $\mathrm{C} \underline{\mathrm{H}}(\mathrm{Ph}) \mathrm{NH}), 4.78(1 \mathrm{H}, \mathrm{d}, J=8.6 \overline{\mathrm{Hz}}, \mathrm{CH}(\mathrm{Ph}) \mathrm{NH}), 6.72(2 \mathrm{H}, \mathrm{d}$, $\left.J=9.0 \mathrm{~Hz}, \mathrm{SO}_{2} \mathrm{Ar}-\underline{\mathrm{H}}\right), 6.86-7.11(10 \mathrm{H}, \overline{\mathrm{m}}, 2 \times \mathrm{Ph}-\underline{\mathrm{H}}), 7.39(2 \mathrm{H}$, $\left.\mathrm{d}, J=9.0 \mathrm{~Hz}, \mathrm{SO}_{2} \mathrm{Ar}-\underline{\mathrm{H}}\right) .{ }^{13} \mathrm{C}-\mathrm{NMR}\left(\mathrm{CD}_{3} \mathrm{OD}\right) \delta: 13.9,20.5,20.7$, $25.5,26.9,28.7,51.2,61.0,64.1,64.8,65.2,68.3,80.6,115.3$, $128.17,128.23,128.4,128.6,129.0,129.1,129.9,134.3,139.8$, 141.0, 157.8, 162.9. IR (KBr) $\mathrm{cm}^{-1}: 3364,3198,3008,2920$, 2853, 1697, 1592, 1491, 1317, 1250, 1150, 1088, 698. HR-MS $(\mathrm{FAB}+) \mathrm{m} / z$ : Found, $624.3477(\mathrm{M})^{+}\left(\right.$Calcd for $\mathrm{C}_{35} \mathrm{H}_{50} \mathrm{~N}_{3} \mathrm{O}_{5} \mathrm{~S}^{+}$: 624.3471).

1-[2-[4-[[](1S,2S)-2-[[(1,1-Dimethylethoxy)carbonyl]amino]-1,2-diphenylethyl]amino]sulfonyl]phenoxy]ethyl]3-methyl-1H-imidazolium Chloride (12) Compound 12 was synthesized from $\mathbf{1 0}(57 \mathrm{mg}, 0.107 \mathrm{mmol})$ in the same manner as the synthesis of 7a. Yield: $61 \mathrm{mg}$ (93\%). Yellow amorphous. $[\alpha]_{\mathrm{D}}^{25}-22.42\left(c=1.40, \mathrm{CH}_{3} \mathrm{OH}\right) .{ }^{1} \mathrm{H}-\mathrm{NMR}$ $\left(\mathrm{CD}_{3} \mathrm{OD}\right) \delta: 1.37(9 \mathrm{H}, \mathrm{s}, t-\mathrm{Bu}), 3.91(3 \mathrm{H}, \mathrm{s}, \mathrm{N}-\mathrm{Me}), 4.30(2 \mathrm{H}$, brs, $\left.\mathrm{CH}_{2} \mathrm{O}\right), 4.53(1 \mathrm{H}, \mathrm{d}, J=8.4 \mathrm{~Hz}, \mathrm{CH}(\mathrm{Ph}) \overline{\mathrm{NH}}), 4.61(2 \mathrm{H}$, brs, $\left.\overline{\mathrm{CH}}_{2} \mathrm{~N}\right), 4.80(1 \mathrm{H}, \mathrm{d}, J=8.9 \mathrm{~Hz}, \mathrm{CH}(\overline{\mathrm{Ph}}) \mathrm{NH}), 6.73(2 \mathrm{H}, \mathrm{d}$, $\left.J=8.8 \overline{\mathrm{Hz}}, \mathrm{SO}_{2} \mathrm{Ar}-\underline{\mathrm{H}}\right), 6.87-7.09(10 \mathrm{H}, \overline{\mathrm{m}}, 2 \times \mathrm{Ph}-\underline{\mathrm{H}}), 7.36(2 \mathrm{H}$, $\left.\mathrm{d}, J=8.6 \mathrm{~Hz}, \mathrm{SO}_{2} \mathrm{Ar}-\underline{\mathrm{H}}\right), 7.57(1 \mathrm{H}, \mathrm{s}$, imidazole- -1$), 7.68(1 \mathrm{H}, \mathrm{s}$,

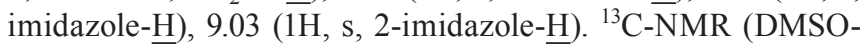
$\left.d_{6}\right) \delta: 28.2,36.0,48.4,59.3,62.2,66.3,78.0,114.4,122.9$, $123.7,126.6,126.7,127.3,127.6,127.8,128.2,134.1,137.3$, $139.5,140.6,155.0,159.9$. IR $(\mathrm{KBr}) \mathrm{cm}^{-1}: 3400,3250,3033$, 2990, 2907, 1697, 1593, 1371, 1247, 1148, 1088, 697. HR-MS $(\mathrm{FAB}+) \mathrm{m} / z$ : Found, $577.2481(\mathrm{M})^{+}\left(\right.$Calcd for $\mathrm{C}_{31} \mathrm{H}_{37} \mathrm{~N}_{4} \mathrm{O}_{5} \mathrm{~S}^{+}$: $577.2485)$.

1-[6-[4-[[](1S,2S)-2-[[(1,1-Dimethylethoxy)carbonyl]amino]-1,2-diphenylethyl]amino]sulfonyl]phenoxy]hexyl]3-methyl-1H-imidazolium Chloride (13) Compound 13 was synthesized from 11 (42 $\mathrm{mg}, 0.071 \mathrm{mmol})$ in the same manner as the synthesis of 7a. Yield: $45 \mathrm{mg}$ (94\%). Yellow 
oil. $[\alpha]_{\mathrm{D}}^{25}-16.46\left(c=1.30, \mathrm{CH}_{3} \mathrm{OH}\right) .{ }^{1} \mathrm{H}-\mathrm{NMR}\left(\mathrm{CD}_{3} \mathrm{OD}\right) \delta$ : 1.28-1.43 (11H, m, $t$-Bu and $\left.\mathrm{CH}_{2}\right), 1.47-1.54\left(2 \mathrm{H}, \mathrm{m}, \mathrm{CH}_{2}\right)$, 1.71-1.78 (2H, m, $\left.\mathrm{CH}_{2}\right), 1.86-1.94\left(2 \mathrm{H}, \mathrm{m}, \mathrm{CH}_{2}\right), 3.89(3 \mathrm{H}, \mathrm{s}$, $\mathrm{N}-\mathrm{Me}), 3.92\left(2 \mathrm{H}, \mathrm{t}, J=6.4 \mathrm{~Hz}, \mathrm{CH}_{2} \mathrm{O}\right), 4.20(2 \mathrm{H}, \mathrm{t}, J=7.3 \mathrm{~Hz}$, $\left.\mathrm{CH}_{2} \mathrm{~N}\right), 4.53(1 \mathrm{H}, \mathrm{d}, J=8.2 \mathrm{~Hz}, \mathrm{CH}(\mathrm{Ph}) \mathrm{NH}), 4.79(1 \mathrm{H}, \mathrm{d}$, $J=8.2 \mathrm{~Hz}, \quad \mathrm{CH}(\mathrm{Ph}) \mathrm{NH}), 6.67\left(2 \mathrm{H}, \overline{\mathrm{d}}, J=9.0 \mathrm{~Hz}, \mathrm{SO}_{2} \mathrm{Ar}-\underline{\mathrm{H}}\right)$, 6.87-7.12 (10) $\overline{\mathrm{H}}, \mathrm{m}, 2 \times \mathrm{Ph}-\underline{\mathrm{H}}), 7.37\left(2 \mathrm{H}, \mathrm{d}, J=8.8 \mathrm{~Hz}, \mathrm{SO}_{2} \overline{\mathrm{Ar}}-\right.$ $\underline{\mathrm{H}}), 7.53(1 \mathrm{H}, \mathrm{d}, J=1.8 \mathrm{~Hz}$, imidazole- $\underline{\mathrm{H}}), 7.61(1 \mathrm{H}, \mathrm{d}, J=1.8 \mathrm{~Hz}$, imidazole- $\mathrm{H}), 8.92\left(1 \mathrm{H}, \mathrm{s}, 2\right.$-imidazole-H). ${ }^{13} \mathrm{C}-\mathrm{NMR}\left(\mathrm{CD}_{3} \mathrm{OD}\right)$ $\delta: 26.5,26.9,28.7,29.8,31.0,36.5,50.7,61.1,64.1,69.1,80.6$, $115.3,123.60,123.64,124.9,125.0,128.1,128.2,128.4,128.6$, $129.0,129.1,129.9,133.9,137.8,139.8,141.0,157.8,163.2$. IR (KBr) $\mathrm{cm}^{-1}:$ 3374, 3207, 3037, 2913, 1694, 1592, 1492, 1318, 1250, 1149, 1088, 716, 580, 554. HR-MS (FAB+) $m / z$ : Found, $633.3108(\mathrm{M})^{+}\left(\right.$Calcd for $\mathrm{C}_{35} \mathrm{H}_{45} \mathrm{~N}_{4} \mathrm{O}_{5} \mathrm{~S}^{+}$: 633.3111).

1-[4-[4-[[](1S,2S)-2-A mino-1,2-diphenylethyl]amino]sulfonyl]phenoxy]butyl]-3-methyl-1 $H$-imidazolium Mono(trifluoroacetate) Salt with Trifluoroacetic Acid (3a) TFA $(0.306 \mathrm{~mL}, 4.12 \mathrm{mmol})$ was added to $7 \mathbf{a}(264 \mathrm{mg}$, $0.412 \mathrm{mmol}$ ) at $0^{\circ} \mathrm{C}$ under $\mathrm{N}_{2}$, and the mixture was stirred for $3.5 \mathrm{~h}$. The volatile material was removed under reduced pressure. Toluene $(5 \mathrm{~mL})$ was added to the residue, and the volatile material was removed $(\times 3)$ to obtain compound 3a. Yield: $299 \mathrm{mg}(99 \%)$. Yellow oil. $[\alpha]_{\mathrm{D}}^{25}-41.60\left(c=0.50, \mathrm{CH}_{3} \mathrm{OH}\right)$. ${ }^{1} \mathrm{H}-\mathrm{NMR}\left(\mathrm{CD}_{3} \mathrm{OD}\right) \quad \delta: 1.73-1.80 \quad\left(2 \mathrm{H}, \mathrm{m}, \mathrm{CH}_{2}\right), 1.98-2.07$ $\left(2 \mathrm{H}, \mathrm{m}, \mathrm{CH}_{2}\right), 3.90(3 \mathrm{H}, \mathrm{s}, \mathrm{N}-\mathrm{Me}), 3.95(2 \mathrm{H}, \mathrm{t}, J=6.0 \mathrm{~Hz}$, $\left.\mathrm{C}_{2} \mathrm{O}\right), 4.27\left(2 \mathrm{H}, \mathrm{t}, J=7.3 \mathrm{~Hz}, \underline{\mathrm{C}}_{2} \mathrm{~N}\right), 4.53(1 \mathrm{H}, \mathrm{d}, J=10.8 \mathrm{~Hz}$, C $\underline{\bar{H}}(\mathrm{Ph}) \mathrm{NH}), 4.66(1 \mathrm{H}, \mathrm{d}, J=10.8 \overline{\mathrm{Hz}}, \mathrm{CH}(\mathrm{Ph}) \mathrm{NH}), 6.69(2 \mathrm{H}, \mathrm{d}$, $\left.J=8.8 \mathrm{~Hz}, \mathrm{SO}_{2} \mathrm{Ar}-\underline{\mathrm{H}}\right), 6.75-7.22(10 \mathrm{H}, \mathrm{m}, 2 \times \mathrm{Ph}-\underline{\mathrm{H}}), 7.47(2 \mathrm{H}, \mathrm{d}$, $\left.J=9.0 \mathrm{~Hz}, \mathrm{SO}_{2} \mathrm{Ar}-\overline{\mathrm{H}}\right), 7.54(1 \mathrm{H}, \mathrm{d}, J=1.8 \mathrm{~Hz}$, imidazole- $\underline{\mathrm{H}}), 7.63$ $(1 \mathrm{H}, \mathrm{d}, J=1.8 \mathrm{~Hz}$, imidazole- $\underline{\mathrm{H}}), 8.97(1 \mathrm{H}, \mathrm{s}, 2$-imidazole- $-\underline{\mathrm{H}})$. ${ }^{13} \mathrm{C}-\mathrm{NMR}\left(\mathrm{CD}_{3} \mathrm{OD}\right) \delta: 26.7, \overline{27.9}, 36.5,50.4,60.7,63.0,6 \overline{8.6}$, $115.4,123.6,125.0,128.7,128.8,129.1,129.2,129.9,130.2$, $130.3,133.2,134.8,136.7,137.9,163.3 .{ }^{19} \mathrm{~F}-\mathrm{NMR}\left(\mathrm{CD}_{3} \mathrm{OD}\right)$ $\delta: 1.80$ (s). IR (KBr) cm $\mathrm{cm}^{-1}$ 3364, 3046, 2910, 1671, 1197, 1153. HR-MS (FAB + ) $m / z$ : Found, $505.2277(\mathrm{M})^{+}$(Calcd for $\mathrm{C}_{28} \mathrm{H}_{33} \mathrm{~N}_{4} \mathrm{O}_{3} \mathrm{~S}^{+}:$505.2273). HR-MS (CI-, isobutane) $\mathrm{m} / \mathrm{z}$ : Found, $112.9855(\mathrm{X})^{-}$(Calcd for $\mathrm{C}_{2} \mathrm{~F}_{3} \mathrm{O}_{2}^{-}$: 112.9850). MS (CI) $\mathrm{m} / z$ (rel. int. \%): $113\left(\mathrm{X}^{-}, 100\right)$. Anal. Calcd for $\mathrm{C}_{32} \mathrm{H}_{34} \mathrm{~F}_{6} \mathrm{~N}_{4} \mathrm{O}_{7} \mathrm{~S} \cdot 5 / 2 \mathrm{H}_{2} \mathrm{O}$ : C, 49.42; H, 5.05; N, 7.20. Found: C, 49.45; H, 4.82; N, 6.85.

1-[4-[4-[[[(1S,2S)-2-Amino-1,2-diphenylethyl]amino]sulfonyl]phenoxy]butyl]-2,3-dimethyl- $1 H$-imidazolium Mono(trifluoroacetate) Salt with Trifluoroacetic Acid (3b) Compound 3b was synthesized from $7 \mathbf{b}(60 \mathrm{mg}, 0.091 \mathrm{mmol})$ in the same manner as the synthesis of 3a. Yield: $64 \mathrm{mg}(95 \%)$. Yellow oil. $[\alpha]_{\mathrm{D}}^{24}-34.26\left(c=1.36, \mathrm{CH}_{3} \mathrm{OH}\right) .{ }^{1} \mathrm{H}-\mathrm{NMR}\left(\mathrm{CD}_{3} \mathrm{OD}\right)$ $\delta: 1.77-1.83\left(2 \mathrm{H}, \mathrm{m}, \mathrm{CH}_{2}\right), 1.92-1.98\left(2 \mathrm{H}, \mathrm{m}, \mathrm{CH}_{2}\right), 2.61(3 \mathrm{H}$, s, 2-imidazole-Me $), 3.79(3 \mathrm{H}, \mathrm{s}, \mathrm{N}-\mathrm{Me}), 3.96(2 \mathrm{H}, \mathrm{t}, J=6.0 \mathrm{~Hz}$, $\left.\mathrm{CH}_{2} \mathrm{O}\right), 4.20\left(2 \overline{\mathrm{H}}, \mathrm{t}, J=7.4 \mathrm{~Hz}, \mathrm{CH}_{2} \mathrm{~N}\right), 4.51(1 \mathrm{H}, \mathrm{d}, J=11.0 \mathrm{~Hz}$, C $\underline{\mathrm{H}}(\mathrm{Ph}) \mathrm{NH}), 4.65(1 \mathrm{H}, \mathrm{d}, J=10.8 \mathrm{~Hz}, \mathrm{CH}(\mathrm{Ph}) \mathrm{NH}), 6.70(2 \mathrm{H}, \mathrm{d}$, $\left.J=9.0 \mathrm{~Hz}, \mathrm{SO}_{2} \mathrm{Ar}-\underline{\mathrm{H}}\right), 6.73-7.41(10 \mathrm{H}, \overline{\mathrm{m}}, 2 \times \mathrm{Ph}-\underline{\mathrm{H}}), 7.45(1 \mathrm{H}$, $\mathrm{d}, J=1.8 \mathrm{~Hz}$, imidazole- $\underline{\mathrm{H}}), 7.47\left(2 \mathrm{H}, \mathrm{d}, J=8.8 \mathrm{~Hz}, \mathrm{SO}_{2} \mathrm{Ar}-\underline{\mathrm{H}}\right)$, $7.51(1 \mathrm{H}, \mathrm{d}, J=1.8 \mathrm{~Hz}$, imidazole- $-\mathrm{H}) .{ }^{13} \mathrm{C}-\mathrm{NMR}\left(\mathrm{CD}_{3} \mathrm{OD}\right) \delta$ : 9.5, 26.8, 27.5, 35.4, 49.1, 60.7, 63.0, 68.7, 115.4, 122.1, 123.7, $128.8,128.9,129.18,129.24,130.0,130.29,130.34,133.3$, 134.9, 136.7, 145.8, 163.3. ${ }^{19} \mathrm{~F}-\mathrm{NMR}\left(\mathrm{CD}_{3} \mathrm{OD}\right) \delta$ : 1.50 (s). IR $(\mathrm{KBr}) \mathrm{cm}^{-1}: 3350,3317,3060,2910,2870,1769,1731,1680$, 1672, 1592, 1494, 1255, 1198, 1167, 600. HR-MS (FAB+) $\mathrm{m} / \mathrm{z}$ : Found, $519.2437(\mathrm{M})^{+}$(Calcd for $\mathrm{C}_{29} \mathrm{H}_{35} \mathrm{~N}_{4} \mathrm{O}_{3} \mathrm{~S}^{+}$: 519.2430). HR-MS (CI-, isobutane) $\mathrm{m} / \mathrm{z}$ : Found, $112.9853(\mathrm{X})^{-}$(Calcd for $\mathrm{C}_{2} \mathrm{~F}_{3} \mathrm{O}_{2}^{-}$: 112.9850). MS (CI) $m / z$ (rel. int. \%): $113\left(\mathrm{X}^{-}, 100\right)$.
Anal. Calcd for $\mathrm{C}_{33} \mathrm{H}_{36} \mathrm{~F}_{6} \mathrm{~N}_{4} \mathrm{O}_{7} \mathrm{~S} \cdot 3 / 2 \mathrm{H}_{2} \mathrm{O}: \mathrm{C}, 51.23 ; \mathrm{H}, 5.08 ; \mathrm{N}$, 7.24. Found: C, 51.06; H, 5.28; N, 7.37.

1-[4-[4-[[[(1S,2S)-2-Amino-1,2-diphenylethyl]amino]sulfonyl]phenoxy]butyl]-pyridinium Mono(trifluoroacetate) Salt with Trifluoroacetic Acid (3c) Compound 3c was synthesized from $7 \mathbf{c}(125 \mathrm{mg}, 0.196 \mathrm{mmol})$ in the same manner as the synthesis of 3a. Yield: $140 \mathrm{mg}(96 \%)$. Yellow amorphous. $[\alpha]_{\mathrm{D}}^{25}-22.14\left(c=1.40, \mathrm{CH}_{3} \mathrm{OH}\right) .{ }^{1} \mathrm{H}-\mathrm{NMR}\left(\mathrm{CD}_{3} \mathrm{OD}\right) \delta$ : 1.79-1.86 (2H, m, $\left.\mathrm{CH}_{2}\right), 2.13-2.21\left(2 \mathrm{H}, \mathrm{m}, \mathrm{CH}_{2}\right), 3.96(2 \mathrm{H}, \mathrm{t}$, $\left.J=6.1 \mathrm{~Hz}, \mathrm{CH}_{2} \mathrm{O}\right), 4.53(1 \mathrm{H}, \mathrm{d}, J=11.0 \mathrm{~Hz}, \underline{\mathrm{CH}}(\mathrm{Ph}) \mathrm{NH}), 4.67$ $(1 \mathrm{H}, \mathrm{d}, J=11.4 \mathrm{~Hz}, \mathrm{CH}(\mathrm{Ph}) \mathrm{NH}), 4.71\left(2 \mathrm{H}, \mathrm{t}, J=7.8 \mathrm{~Hz}, \mathrm{CH}_{2} \mathrm{~N}\right)$, $6.69\left(2 \mathrm{H}, \mathrm{d}, J=9.0 \mathrm{~Hz}, \mathrm{SO}_{2} \mathrm{Ar}-\underline{\mathrm{H}}\right), 6.75-7.29(10 \mathrm{H}, \mathrm{m}, 2 \times \mathrm{Ph}-$ $\underline{\mathrm{H}}), 7.47\left(2 \mathrm{H}, \mathrm{d}, J=9.0 \mathrm{~Hz}, \mathrm{SO}_{2} \mathrm{Ar}-\underline{\mathrm{H}}\right), 8.10(2 \mathrm{H}, \mathrm{t}, J=7.1 \mathrm{~Hz}$, 3,5-pyridine- $\underline{\mathrm{H}}), 8.57$ (1H, t, $J=7.8 \mathrm{~Hz}, 4$-pyridine- $\underline{\mathrm{H}}), 9.03$ $\left(2 \mathrm{H}, \mathrm{d}, J=5.5 \mathrm{~Hz}, 2,6\right.$-pyridine-H). ${ }^{13} \mathrm{C}-\mathrm{NMR}\left(\mathrm{CD}_{3} \mathrm{OD}\right) \delta: 26.6$, $29.2,60.6,62.7,63.0,68.5,115.4,128.7,128.8,129.15,129.21$, $129.3,129.5,129.9,130.18,130.24,130.3,133.3,134.9,136.7$, 145.9, 146.9, 163.2. ${ }^{19} \mathrm{~F}-\mathrm{NMR}\left(\mathrm{CD}_{3} \mathrm{OD}\right) \delta$ : 1.85 (s). IR (KBr) $\mathrm{cm}^{-1}:$ 3379, 3042, 2913, 2671, 1680, 1629, 1606, 1198, 1175, 1149, 1126, 1092, 698. HR-MS (FAB+) $m / z$ : Found, 502.2159 $(\mathrm{M})^{+}$(Calcd for $\mathrm{C}_{29} \mathrm{H}_{32} \mathrm{~N}_{3} \mathrm{O}_{3} \mathrm{~S}^{+}$: 502.2164). HR-MS (CI-, isobutane) $m / z$ : Found, $112.9842(\mathrm{X})^{-}$(Calcd for $\mathrm{C}_{2} \mathrm{~F}_{3} \mathrm{O}_{2}{ }^{-}$: 112.9850). MS (CI) $m / z$ (rel. int. \%): 113 (X', 100). Anal. Calcd for $\mathrm{C}_{33} \mathrm{H}_{33} \mathrm{~F}_{6} \mathrm{~N}_{3} \mathrm{O}_{7} \mathrm{~S} \cdot 1 / 2 \mathrm{H}_{2} \mathrm{O}$ : C, 53.66; H, 4.64; N, 5.69. Found: C, 53.67; H, 4.75; N, 5.77.

1-[4-[4-[[[(1S,2S)-2-Amino-1,2-diphenylethyl]amino]sulfonyl]phenoxy]butyl]-3-methylbenzimidazolium Mono(trifluoroacetate) Salt with Trifluoroacetic Acid (3d) Compound 3d was synthesized from 7d (94 mg, $0.136 \mathrm{mmol})$ in the same manner as the synthesis of 3a. Yield: $108 \mathrm{mg}$ (99\%). Yellow oil. $[\alpha]_{\mathrm{D}}^{25}-35.93\left(c=1.92, \mathrm{CH}_{3} \mathrm{OH}\right) .{ }^{1} \mathrm{H}-\mathrm{NMR}$ $\left(\mathrm{CD}_{3} \mathrm{OD}\right) \delta: 1.84-1.89\left(2 \mathrm{H}, \mathrm{m}, \mathrm{CH}_{2}\right), 2.12-2.19\left(2 \mathrm{H}, \mathrm{m}, \mathrm{CH}_{2}\right)$, $3.97\left(3 \mathrm{H}, \mathrm{t}, J=6.0 \mathrm{~Hz}, \mathrm{CH}_{2} \mathrm{O}\right), 4.12(3 \mathrm{H}, \mathrm{s}, \mathrm{N}-\mathrm{Me}), 4.51 \overline{(1 \mathrm{H}}$, $\mathrm{d}, J=11.0 \mathrm{~Hz}, \mathrm{CH}(\mathrm{Ph}) \mathrm{NH}), 4.58\left(2 \mathrm{H}, \mathrm{t}, J=7.3 \mathrm{~Hz}, \mathrm{CH}_{2} \mathrm{~N}\right), 4.65$ $(1 \mathrm{H}, \mathrm{d}, J=11.0 \mathrm{~Hz}, \mathrm{CH}(\mathrm{Ph}) \mathrm{NH}), 6.66\left(2 \mathrm{H}, \mathrm{d}, J=8.8 \mathrm{~Hz}, \mathrm{SO}_{2} \mathrm{Ar}-\right.$ $\underline{\mathrm{H}}), 6.73-7.22(10 \mathrm{H}, \mathrm{m}, 2 \times \mathrm{Ph}-\underline{\mathrm{H}}), 7.44(2 \mathrm{H}, \mathrm{d}, J=9.0 \mathrm{~Hz}$, $\left.\mathrm{SO}_{2} \mathrm{Ar}-\underline{\mathrm{H}}\right), \quad 7.69-7.72$ (2H, m, benzimidazole- $\left.\underline{\mathrm{H}}\right), \quad 7.90-7.98$ ( $2 \mathrm{H}, \mathrm{m}$, benzimidazole- $\underline{\mathrm{H}}), 9.53$ (1H, s, 2-benzimidazole- $\underline{\mathrm{H}})$. ${ }^{13} \mathrm{C}-\mathrm{NMR}\left(\mathrm{CD}_{3} \mathrm{OD}\right) \delta:$ 26.9, 27.0, 33.8, 48.1, 60.7, 63.0, 68.5, $114.30,114.34,115.3,128.18,128.20,128.7,128.8,129.15$, $129.22,129.9,130.2,130.3,132.7,133.2,133.6,134.9,136.7$, 143.4, 163.2. ${ }^{19} \mathrm{~F}-\mathrm{NMR}\left(\mathrm{CD}_{3} \mathrm{OD}\right) \delta: 1.34$ (s). IR (KBr) $\mathrm{cm}^{-1}$ : 3372, 3127, 3080, 2940, 2861, 1690, 1670, 1595, 1570, 1497, 1255, 1198, 1175, 1149, 700. HR-MS (FAB+) $m / z$ : Found, $555.2433(\mathrm{M})^{+}$(Calcd for $\mathrm{C}_{32} \mathrm{H}_{35} \mathrm{~N}_{4} \mathrm{O}_{3} \mathrm{~S}^{+}$: 555.2430). HR-MS (CI-, isobutane) $\mathrm{m} / \mathrm{z}$ : Found, $112.9842(\mathrm{X})^{-}$(Calcd for $\mathrm{C}_{2} \mathrm{~F}_{3} \mathrm{O}_{2}{ }^{-}$: 112.9850). MS (CI) $m / z$ (rel. int. \%): 113 (X', 100). Anal. Calcd for $\mathrm{C}_{36} \mathrm{H}_{36} \mathrm{~F}_{6} \mathrm{~N}_{4} \mathrm{O}_{7} \mathrm{~S} \cdot 4 / 3 \mathrm{H}_{2} \mathrm{O}: \mathrm{C}, 53.59 ; \mathrm{H}, 4.83 ; \mathrm{N}, 6.94$. Found: C, 53.78; H, 4.92; N, 6.73.

1 - [4 - [4 - [ [ [ $(1 S, 2 S)$-2 - A m in o-1, 2 - d iphenylet hyl] amino]sulfonyl]phenoxy]butyl]-1-methylpy rolidinium Mono(trifluoroacetate) Salt with Trifluoroacetic Acid (3e) Compound 3e was synthesized from 7 e $(60 \mathrm{mg}, 0.093 \mathrm{mmol})$ in the same manner as the synthesis of 3a. Yield: $61 \mathrm{mg}$ (92\%). Yellow amorphous. $[\alpha]_{\mathrm{D}}^{25}-50.40\left(c=1.00, \mathrm{CH}_{3} \mathrm{OH}\right)$. ${ }^{1} \mathrm{H}-\mathrm{NMR} \quad\left(\mathrm{CD}_{3} \mathrm{OD}\right) \quad \delta: 1.78-1.85 \quad\left(2 \mathrm{H}, \mathrm{m}, \mathrm{CH}_{2}\right), 1.91-1.99$ $\left(2 \mathrm{H}, \mathrm{m}, \mathrm{CH}_{2}\right), 2.21$ (4H, brs, 3,4-pyrrolidine- $\left.{ }_{\mathrm{H}}\right), 3.06$ (3H, s, $\mathrm{N}-\mathrm{Me}), 3.41\left(2 \mathrm{H}\right.$, brt, $\left.J=8.4 \mathrm{~Hz}, \mathrm{CH}_{2} \mathrm{~N}\right), 3.47-3.58(4 \mathrm{H}, \mathrm{m}$, 2,5-pyrrolidine- $\underline{\mathrm{H}}), 3.99\left(2 \mathrm{H}, \mathrm{t}, J=6.0 \mathrm{~Hz}, \underline{\mathrm{CH}}_{2} \mathrm{O}\right), 4.52(1 \mathrm{H}, \mathrm{d}$, $J=10.8 \mathrm{~Hz}, \mathrm{CH}(\overline{\mathrm{Ph}}) \mathrm{NH}), 4.65(1 \mathrm{H}, \mathrm{d}, J=10 . \overline{\mathrm{Hz}}, \mathrm{CH}(\mathrm{Ph}) \mathrm{NH})$, $6.73\left(2 \mathrm{H}, \mathrm{d}, \bar{J}=9.0 \mathrm{~Hz}, \mathrm{SO}_{2} \mathrm{Ar}-\underline{\mathrm{H}}\right), 6.75-7.22(10 \mathrm{H}, \overline{\mathrm{m}}, 2 \times \mathrm{Ph}-$ 
$\mathrm{H}), 7.49\left(2 \mathrm{H}, \mathrm{d}, J=9.0 \mathrm{~Hz}, \mathrm{SO}_{2} \mathrm{Ar}-\mathrm{H}\right) .{ }^{13} \mathrm{C}-\mathrm{NMR}\left(\mathrm{CD}_{3} \mathrm{OD}\right) \delta$ : 21.7, 22.5, 27.0, 60.7, 63.0, 65.1, 65.4, 68.5, 115.4, 128.8, 128.9, $129.2,129.3,130.0,130.29,130.33,133.4,134.9,136.7,163.3$ (one aliphatic peak could not be found). ${ }^{19} \mathrm{~F}-\mathrm{NMR}\left(\mathrm{CD}_{3} \mathrm{OD}\right)$ $\delta$ : 1.68 (s). IR (KBr) cm $\mathrm{cm}^{-1}$ : 3380, 3042, 2928, 2860, 2658, 1700, 1680, 1593, 1498, 1250, 1150, 696. HR-MS (FAB+) $m / z$ : Found, $508.2638(\mathrm{M})^{+}$(Calcd for $\mathrm{C}_{29} \mathrm{H}_{38} \mathrm{~N}_{3} \mathrm{O}_{3} \mathrm{~S}^{+}$: 508.2634). HR-MS (CI-, isobutane) $\mathrm{m} / \mathrm{z}$ : Found, $112.9858(\mathrm{X})^{-}$(Calcd for $\mathrm{C}_{2} \mathrm{~F}_{3} \mathrm{O}_{2}^{-}$: 112.9850). MS (CI) $m / z$ (rel. int. \%): $113\left(\mathrm{X}^{-}, 100\right)$. Anal. Calcd for $\mathrm{C}_{33} \mathrm{H}_{39} \mathrm{~F}_{6} \mathrm{~N}_{3} \mathrm{O}_{7} \mathrm{~S} \cdot 1 / 2 \mathrm{H}_{2} \mathrm{O}: \mathrm{C}, 52.58 ; \mathrm{H}, 5.48 ; \mathrm{N}$, 5.57. Found: C, 52.87; H, 5.64; N, 5.62.

1-[4-[4-[[[(1S,2S)-2-Amino-1,2-diphenylethyl]amino]sulfonyl]phenoxy]butyl]-1- $N$-butyl- $N, N$-dimethyl-ammonium Mono(trifluoroacetate) Salt with Trifluoroacetic Acid (3f) Compound 3f was synthesized from 7 f $(52 \mathrm{mg}, 0.079 \mathrm{mmol})$ in the same manner as the synthesis of 3a. Yield: $59 \mathrm{mg}$ (99\%). Yellow oil. $[\alpha]_{\mathrm{D}}^{25}-38.80\left(c=1.00, \mathrm{CH}_{3} \mathrm{OH}\right) .{ }^{1} \mathrm{H}-\mathrm{NMR}$ $\left(\mathrm{CD}_{3} \mathrm{OD}\right) \delta: 0.98\left(3 \mathrm{H}, \mathrm{t}, J=7.3 \mathrm{~Hz}, \mathrm{CH}_{3}\right), 1.34-1.43(2 \mathrm{H}$, $\left.\mathrm{m}, \mathrm{CH}_{2}\right), 1.67-1.75\left(2 \mathrm{H}, \mathrm{m}, \mathrm{CH}_{2}\right), 1.77-1.84\left(2 \mathrm{H}, \mathrm{m}, \mathrm{CH}_{2}\right)$, 1.87-1.95 (2H, m, $\left.\mathrm{CH}_{2}\right), 3.06(6 \mathrm{H}, \mathrm{s}, 2 \times \mathrm{N}-\mathrm{Me}), 3.29(2 \mathrm{H}, \mathrm{t}$, $\left.J=8.4 \mathrm{~Hz}, \mathrm{CH}_{2} \mathrm{~N}\right), 3.35\left(2 \mathrm{H}\right.$, brt, $\left.J=8.4 \mathrm{~Hz}, \overline{\mathrm{CH}}_{2} \mathrm{~N}\right), 3.99(2 \mathrm{H}$, t, $\left.J=5.9 \mathrm{~Hz}, \underline{\mathrm{C}}_{2} \mathrm{O}\right), 4.49(1 \mathrm{H}, \mathrm{d}, J=10.8 \mathrm{~Hz}, \underline{\mathrm{C}} \underline{\mathrm{H}}(\mathrm{Ph}) \mathrm{NH}), 4.63$ $(1 \mathrm{H}, \mathrm{d}, J=10.8 \overline{\mathrm{Hz}}, \mathrm{CH}(\mathrm{Ph}) \mathrm{NH}), 6.73\left(2 \mathrm{H}, \mathrm{d}, J=9.0 \mathrm{~Hz}, \mathrm{SO}_{2} \mathrm{Ar}-\right.$ $\underline{\mathrm{H}}), 6.86-7.22(10 \mathrm{H}, \mathrm{m}, 2 \times \mathrm{Ph}-\underline{\mathrm{H}}), 7.49(2 \mathrm{H}, \mathrm{d}, J=8.8 \mathrm{~Hz}$, $\left.\mathrm{SO}_{2} \mathrm{Ar}-\underline{\mathrm{H}}\right) .{ }^{13} \mathrm{C}-\mathrm{NMR}\left(\mathrm{CD}_{3} \mathrm{OD}\right) \delta: 13.9,20.4,20.6,25.4,26.8$, $51.2,60.7,63.0,64.8,65.2,68.4,115.4,128.7,128.9,129.1$, $129.3,130.0,130.3,130.4,133.4,134.9,136.7,163.3 .{ }^{19} \mathrm{~F}-\mathrm{NMR}$ $\left(\mathrm{CD}_{3} \mathrm{OD}\right) \delta: 1.69$ (s). IR (KBr) cm $\mathrm{cm}^{-1}: 3376,3042,2970,2860$, $1668,1593,1492,1255,1196,1152,1128,700$. HR-MS $(\mathrm{FAB}+) \mathrm{m} / \mathrm{z}$ : Found, $524.2943(\mathrm{M})^{+}\left(\right.$Calcd for $\mathrm{C}_{30} \mathrm{H}_{42} \mathrm{~N}_{3} \mathrm{O}_{3} \mathrm{~S}^{+}$: 524.2947). HR-MS (CI-, isobutane) $m / z$ : Found, $112.9843(\mathrm{X})^{-}$ (Calcd for $\mathrm{C}_{2} \mathrm{~F}_{3} \mathrm{O}_{2}^{-}$: 112.9850). MS (CI) $\mathrm{m} / z$ (rel. int. \%): 113 (X, 100). Anal. Calcd for $\mathrm{C}_{34} \mathrm{H}_{43} \mathrm{~F}_{6} \mathrm{~N}_{3} \mathrm{O}_{7} \mathrm{~S} \cdot 3 / 2 \mathrm{H}_{2} \mathrm{O}: \mathrm{C}, 52.44$; H, 5.95; N, 5.40. Found: C, 52.68; H, 5.97; N, 5.25.

1-[2-[4-[[](1S,2S)-2-Amino-1,2-diphenylethyl]amino]sulfonyl]phenoxy]butyl]-3-methyl-1H-imidazolium Mono(trifluoroacetate) Salt with Trifluoroacetic Acid (14) Compound 14 was synthesized from 12 (50 mg, $0.082 \mathrm{mmol})$ in the same manner as the synthesis of 3a. Yield: $57 \mathrm{mg}$ (99\%). Yellow oil. $[\alpha]_{\mathrm{D}}^{25}-46.60\left(c=1.06, \mathrm{CH}_{3} \mathrm{OH}\right) .{ }^{1} \mathrm{H}-\mathrm{NMR}$ $\left(\mathrm{CD}_{3} \mathrm{OD}\right) \delta: 3.93(3 \mathrm{H}, \mathrm{s}, \mathrm{N}-\underline{\mathrm{Me}}), 4.30\left(2 \mathrm{H}, \mathrm{t}, J=4.6 \mathrm{~Hz}, \underline{\mathrm{CH}}_{2} \mathrm{O}\right)$, $4.47(1 \mathrm{H}, \mathrm{d}, J=11.0 \mathrm{~Hz}, \mathrm{C} \underline{\mathrm{H}}(\mathrm{Ph}) \mathrm{NH}), 4.60-4.63\left(3 \mathrm{H}, \mathrm{m}, \overline{\mathrm{CH}}_{2} \mathrm{~N}\right.$ and $\mathrm{CH}(\mathrm{Ph}) \mathrm{NH}), 6.71-7.22(10 \mathrm{H}, \mathrm{m}, 2 \times \mathrm{Ph}-\mathrm{H}), 6.77(2 \mathrm{H}, \mathrm{d}$, $\left.J=8.8 \overline{\mathrm{Hz}}, \mathrm{SO}_{2} \mathrm{Ar}-\underline{\mathrm{H}}\right), 7.49\left(2 \mathrm{H}, \mathrm{d}, J=8.8 \mathrm{~Hz}, \mathrm{SO}_{2} \mathrm{Ar}-\underline{\mathrm{H}}\right), 7.58$ $(1 \mathrm{H}, \mathrm{s}$, imidazole- $\underline{\mathrm{H}}), 7.68(1 \mathrm{H}, \mathrm{s}$, imidazole- $\underline{\mathrm{H}}), 9.02(1 \mathrm{H}, \mathrm{s}$, 2-imidazole- $-{ }^{13}$ C-NMR ( $\left.{ }^{13} D_{3} \mathrm{OD}\right) \delta: 36.6, \overline{50.1}, 60.7,63.0$, $67.5,115.6,124.2,125.0,128.77,128.84,129.16,129.23,130.0$, $130.4,134.3,134.9,136.7,138.6,162.2 .{ }^{19} \mathrm{~F}-\mathrm{NMR}\left(\mathrm{CD}_{3} \mathrm{OD}\right) \delta$ : 3.13 (brs). IR (KBr) cm $\mathrm{cm}^{-1}$ : 3379, 3049, 2904, 2850, 2646, 1680, 1660, 1595, 1196, 1154, 1131. HR-MS (FAB+) $m / z$ : Found, $477.1967(\mathrm{M})^{+}$(Calcd for $\mathrm{C}_{26} \mathrm{H}_{29} \mathrm{~N}_{4} \mathrm{O}_{3} \mathrm{~S}^{+}$: 477.1960). HR-MS (CI-, isobutane) $\mathrm{m} / \mathrm{z}$ : Found, $112.9855(\mathrm{X})^{-}$(Calcd for $\mathrm{C}_{2} \mathrm{~F}_{3} \mathrm{O}_{2}^{-}$: 112.9850). MS (CI) $m / z$ (rel. int. \%): 113 (X', 100). Anal. Calcd for $\mathrm{C}_{30} \mathrm{H}_{30} \mathrm{~F}_{6} \mathrm{~N}_{4} \mathrm{O}_{7} \mathrm{~S} \cdot 3 / 2 \mathrm{H}_{2} \mathrm{O}$ : C, 49.25; H, 4.55; N, 7.66. Found: C, 49.49; H, 4.88; N, 7.95.

1-[6-[4-[[[(1S,2S)-2-Amino-1,2-diphenylethyl]amino]sulfonyl]phenoxy]hexyl]-3-methyl-1H-imidazolium Mono(trifluoroacetate) Salt with Trifluoroacetic Acid (15) Compound 15 was synthesized from 13 (41 mg, $0.061 \mathrm{mmol})$ in the same manner as the synthesis of 3a. Yield: $45 \mathrm{mg}(97 \%)$. Yellow oil. $[\alpha]_{\mathrm{D}}^{25}-37.70\left(c=0.96, \mathrm{CH}_{3} \mathrm{OH}\right) .{ }^{1} \mathrm{H}-\mathrm{NMR}\left(\mathrm{CD}_{3} \mathrm{OD}\right)$ $\delta: 1.35-1.43\left(2 \mathrm{H}, \mathrm{m}, \mathrm{CH}_{2}\right), 1.46-1.54\left(2 \mathrm{H}, \mathrm{m}, \mathrm{CH}_{2}\right), 1.70-1.77$ $\left(2 \mathrm{H}, \mathrm{m}, \mathrm{CH}_{2}\right), 1.85-1.94\left(2 \mathrm{H}, \mathrm{m}, \mathrm{CH}_{2}\right), 3.89(3 \mathrm{H}, \mathrm{s}, \mathrm{N}-\mathrm{Me})$, $3.91\left(2 \mathrm{H}, \mathrm{t}, J=6.2 \mathrm{~Hz}, \underline{\mathrm{CH}}_{2} \mathrm{O}\right), 4.20\left(2 \mathrm{H}, \mathrm{t}, J=7.3 \mathrm{~Hz}, \mathrm{CH}_{2} \mathrm{~N}\right)$, $4.49(1 \mathrm{H}, \mathrm{d}, J=11.0 \mathrm{~Hz}, \overline{\mathrm{CH}}(\mathrm{Ph}) \mathrm{NH}), 4.62(1 \mathrm{H}, \mathrm{d}, J=11.0 \mathrm{~Hz}$, $\mathrm{CH}(\mathrm{Ph}) \mathrm{NH}), 6.69\left(2 \mathrm{H}, \mathrm{d}, J=9.0 \mathrm{~Hz}, \mathrm{SO}_{2} \mathrm{Ar}-\underline{\mathrm{H}}\right), 6.72-7.22(10 \mathrm{H}$, $\mathrm{m}, 2 \times \mathrm{Ph}-\underline{\mathrm{H}}), 7.46\left(2 \mathrm{H}, \mathrm{d}, J=9.0 \mathrm{~Hz}, \mathrm{SO}_{2}-\underline{\mathrm{Ar}}-\underline{\mathrm{H}}\right), 7.54(1 \mathrm{H}, \mathrm{d}$, $J=1.8 \mathrm{~Hz}$, imidazole- $-\mathrm{H}), 7.62(1 \mathrm{H}, \mathrm{d}, J=2.0 \mathrm{~Hz}$, imidazole$\underline{\mathrm{H}}), 8.93(1 \mathrm{H}, \mathrm{s}, 2$-imidazole- $\underline{\mathrm{H}}) .{ }^{13} \mathrm{C}-\mathrm{NMR}\left(\mathrm{CD}_{3} \mathrm{OD}\right) \delta: 26.4$, 26.9, 29.8, 31.0, 36.4, 50.7, 60.7, 63.0, 69.2, 115.4, 123.6, 123.7, $124.9,125.0,128.8,128.9,129.2,129.3,130.0,130.3,130.4$, 133.0, 134.9, 136.7, 137.9, 163.6. ${ }^{19} \mathrm{~F}-\mathrm{NMR}\left(\mathrm{CD}_{3} \mathrm{OD}\right) \delta: 1.92$ (s). IR (KBr) cm ${ }^{-1}$ : 3410, 3080, 2950, 1682, 1675, 1592, 1199, 1179, 1151, 1129. HR-MS (FAB+) $m / z$ : Found, $533.2591(\mathrm{M})^{+}$ (Calcd for $\mathrm{C}_{30} \mathrm{H}_{37} \mathrm{~N}_{4} \mathrm{O}_{3} \mathrm{~S}^{+}$: 533.2586). HR-MS (CI-, isobutane) $\mathrm{m} / \mathrm{z}$ : Found, $112.9848(\mathrm{X})^{-}$(Calcd for $\mathrm{C}_{2} \mathrm{~F}_{3} \mathrm{O}_{2}{ }^{-}$: 112.9850). MS (CI) $m / z$ (rel. int. \%): $113\left(\mathrm{X}^{-}, 100\right)$. Anal. Calcd for $\mathrm{C}_{34} \mathrm{H}_{38} \mathrm{~F}_{6} \mathrm{~N}_{4} \mathrm{O}_{7} \mathrm{~S} \cdot 2 \mathrm{H}_{2} \mathrm{O}: \mathrm{C}, 51.25 ; \mathrm{H}, 5.31 ; \mathrm{N}, 7.03$. Found: C, 51.59; H, 5.61; N, 6.74.

Typical Procedure of RCATH Acetophenone $(120 \mathrm{mg}$, $1.0 \mathrm{mmol})$ was added to a solution of ionic ligand $(0.012 \mathrm{mmol})$ and $\left[\mathrm{RuCl}_{2} \text { (benzene) }\right]_{2} \quad(2.5 \mathrm{mg}, \quad 0.005 \mathrm{mmol})$ in [bmim] $\left[\mathrm{PF}_{6}\right](1.0 \mathrm{~mL})$ with stirring under $\mathrm{N}_{2}$, followed by addition of a formic acid-triethylamine azeotropic mixture ${ }^{31)}$ (bp $\left.108^{\circ} \mathrm{C} / 29 \mathrm{mmHg}, 0.5 \mathrm{~mL}\right)$. The reaction mixture was stirred at rt for $24 \mathrm{~h}$. Next, $n$-hexane $(5 \mathrm{~mL} \times 3)$ was added to the reaction mixture and the products were extracted by decantation of the upper layer, and the residual IL phase was dried in vacuo for $30 \mathrm{~min}$. Acetophenone $(120 \mathrm{mg}, 1.0 \mathrm{mmol})$ and formic acidtriethylamine azeotropic mixture $(0.5 \mathrm{~mL})$ were added to the remaining IL solution, and the next cycle of the reaction was started.

Acknowledgment A part of this work was financially supported by a Grant-in-Aid for Scientific Research (C) (Research project number: 23590031) from Japan Society for the Promotion of Science (JSPS).

Conflict of Interest The authors declare no conflict of interest.

Supplementary Materials The online version of this article contains supplementary materials.

\footnotetext{
References

1) Palmer M. J., Wills M., Tetrahedron Asymmetry, 10, 2045-2061 (1999).

2) Ohkuma T., Noyori R., "Comprehensive Asymmetric Catalysis I," ed. by Jacobsen E. N., Pfaltz A., Yamamoto H., Springer, Berlin, 1999, pp. 199-246.

3) Hashiguchi S., Fujii A., Takehara J., Ikariya T., Noyori R., J. Am. Chem. Soc., 117, 7562-7563 (1995).

4) Noyori R., Yamakawa M., Hashiguchi S., J. Org. Chem., 66, 79317944 (2001).

5) Noyori R., Angew. Chem. Int. Ed. Engl., 41, 2008-2022 (2002).

6) ter Halle R., Schulz E., Lemaire M., Synlett, 1997, 1257-1258 (1997).

7) Bayston D. J., Travers C. B., Polywka M. E. C., Tetrahedron Asymmetry, 9, 2015-2018 (1998).

8) Li X., Chen W., Hems W., King F., Xiao J., Tetrahedron Lett., 45, 951-953 (2004)

9) Wu X., Li X., Hems W., King F., Xiao J., Org. Biomol. Chem., 2, 1818-1821 (2004).
} 
10) Li X., Wu X., Chen W., Hancock F. E., King F., Xiao J., Org. Lett., 6, 3321-3324 (2004).

11) Chen Y.-C., Wu T.-F., Deng J.-G., Liu H., Jiang Y.-Z., Choi M. C. K., Chan A. S. C., Chem. Commun., 2001, 1488-1489 (2001).

12) Chen Y.-C., Wu T.-F., Deng J.-G., Liu H., Cui X., Zhu J., Jiang Y.-Z., Choi M. C. K., Chan A. S. C., J. Org. Chem., 67, 5301-5306 (2002).

13) Chen Y.-C., Wu T.-F., Jiang L., Deng J.-G., Liu H., Zhu J., Jiang Y.-Z., J. Org. Chem., 70, 1006-1010 (2005).

14) Welton T., Chem. Rev., 99, 2071-2084 (1999).

15) Wasserscheid P., Keim W., Angew. Chem. Int. Ed. Engl., 39, $3772-$ 3789 (2000).

16) Sheldon R., Chem. Commun., 2001, 2399-2407 (2001).

17) Trindade A. F., Gois P. M. P., Afonso C. A. M., Chem. Rev., 109, 418-514 (2009).

18) Ni B., Headley A. D., Chem. Eur. J., 16, 4426-4436 (2010).

19) Huddleston J. G., Visser A. E., Reichert W. M., Willauer H. D., Broker G. A., Rogers R. D., Green Chem., 3, 156-164 (2001).

20) Comyns C., Karodia N., Zeler S., Andersen J.-A., Catal. Lett., 67, $113-115$ (2000).
21) Berthold H., Schotten T., Hönig H., Synthesis, 11, 1607-1610 (2002).

22) Geldbach T. J., Dyson P. J., J. Am. Chem. Soc., 126, 8114-8115 (2004).

23) Baán Z., Finta Z., Keglevich G., Hermecz I., Tetrahedron Lett., 46, 6203-6204 (2005).

24) Joerger J.-M., Paris J.-M., Vaultier M., ARKIVOC, 2006, 152-160 (2006).

25) Baán Z., Finta Z., Keglevich G., Hermecz I., Green Chem., 11, 1937-1940 (2009).

26) Kawasaki I., Tsunoda K., Tsuji T., Yamaguchi T., Shibuta H., Uchida N., Yamashita M., Ohta S., Chem. Commun., 2005, 2134-2136 (2005).

27) Foowler F. W., J. Org. Chem., 37, 1321-1322 (1972).

28) Matsunaga H., Ishizuka T., Kunieda T., Tetrahedron Lett., 46, 3645-3648 (2005).

29) Brown H. C., Chandrasekharan J., Ramachandran P. V., J. Am. Chem. Soc., 110, 1539-1546 (1988).

30) Shinkai S., Minami T., Araragi Y., Manabe O., J. Chem. Soc., Perkin Trans. II, 1985, 503-509 (1985).

31) Narita K., Sekiya M., Chem. Pharm. Bull., 25, 135-140 (1977). 